\title{
MATRIX-VARIATE GAUSS HYPERGEOMETRIC DISTRIBUTION
}

\author{
ARJUN K. GUPTA and DAYA K. NAGAR ${ }^{\bowtie}$ \\ (Received 13 November 2010; accepted 11 April 2012; first published online 4 March 2013) \\ Communicated by V. T. Stefanov
}

\begin{abstract}
In this paper, we propose a matrix-variate generalization of the Gauss hypergeometric distribution and study several of its properties. We also derive probability density functions of the product of two independent random matrices when one of them is Gauss hypergeometric. These densities are expressed in terms of Appell's first hypergeometric function $F_{1}$ and Humbert's confluent hypergeometric function $\boldsymbol{\Phi}_{1}$ of matrix arguments.
\end{abstract}

2010 Mathematics subject classification: primary 62H99; secondary 62E15.

Keywords and phrases: beta distribution, beta function, gamma function, invariant polynomial, matrixvariate, transformation, zonal polynomial.

\section{Introduction}

The random variable $X$ is said to have a Gauss hypergeometric (GH) distribution with parameters $\alpha, \beta, \gamma$ and $\xi$ (where $\alpha>0, \beta>0,-\infty<\gamma<\infty$ and $\xi>-1$ ), denoted by $X \sim \mathrm{GH}(\alpha, \beta, \gamma, \xi)$, if its probability density function (p.d.f.) is given by

$$
\mathrm{GH}(x ; \alpha, \beta, \gamma, \xi)=C(\alpha, \beta, \gamma, \xi) \frac{x^{\alpha-1}(1-x)^{\beta-1}}{(1+\xi x)^{\gamma}},
$$

where $0<x<1$,

$$
\begin{gathered}
C(\alpha, \beta, \gamma, \xi)=\left[\mathrm{B}(\alpha, \beta){ }_{2} F_{1}(\gamma, \alpha ; \alpha+\beta ;-\xi)\right]^{-1}, \\
\mathrm{~B}(\alpha, \beta)=\frac{\Gamma(\alpha) \Gamma(\beta)}{\Gamma(\alpha+\beta)},
\end{gathered}
$$

and ${ }_{2} F_{1}$ is the Gauss hypergeometric function (Luke [14]). This distribution was suggested by Armero and Bayarri [2] in connection with the prior distribution of the

The second author was supported by the Comité para el Desarrollo de la Investigación, Universidad de Antioquia research grant no. IN560CE.

(C) 2013 Australian Mathematical Publishing Association Inc. 1446-7887/2013 \$16.00 
parameter $\rho$ that represents the traffic intensity in an $M / M / 1$ queueing system (here $0<$ $\rho<1$ ). When either $\gamma$ or $\xi$ is equal to zero, the Gauss hypergeometric density reduces to a beta type 1 density (Johnson et al. [12]). Further, if $\gamma=\alpha+\beta$ and $\xi=1$, the Gauss hypergeometric distribution simplifies to a beta type 3 distribution (Cardeño et al. [3]). If $\gamma=\alpha+\beta$ and $\xi=-(1-\lambda)$, the Gauss hypergeometric distribution becomes a threeparameter beta type 1 distribution (Libby and Novic [13], Nadarajah [15], and Nagar and Rada-Mora [17]).

In this paper, we define a matrix-variate generalization of the Gauss hypergeometric distribution and study its properties and relationship with other matrixvariate distributions.

In Section 2 we give some preliminary results on matrix algebra, integration, zonal and invariant polynomials and special functions of matrix argument. Section 3 gives the definition of the matrix-variate Gauss hypergeometric distribution. In Section 4 we study certain properties such as moments, moment generating functions, marginal and conditional distributions. Finally, in Section 5, we derive distributions of certain random quadratic forms involving the matrix-variate Gauss hypergeometric distribution.

\section{Some well-known results and definitions}

We begin with a brief review of some definitions and notation. We adhere to standard notation. Let $A=\left(a_{i j}\right)$ be a symmetric $m \times m$ matrix. Then $A^{T}$ denotes the transpose of $A$ and $\operatorname{tr}(A)$ and $\operatorname{det}(A)$ its trace and determinant; we write $\operatorname{etr}(A)=$ $\exp (\operatorname{tr}(A))$. The norm of $A$, written $\|A\|$, is the maximum of the absolute values of the eigenvalues of $A$. Next, $A>0$ means that $A$ is symmetric positive definite and $A^{1 / 2}$ denotes the unique symmetric positive definite square root of $A$. The multivariate gamma function, for $\operatorname{Re}(a)>(m-1) / 2$, is defined by

$$
\begin{aligned}
\Gamma_{m}(a) & =\int_{X>0} \operatorname{etr}(-X) \operatorname{det}(X)^{a-(m+1) / 2} d X \\
& =\pi^{m(m-1) / 4} \prod_{i=1}^{m} \Gamma\left(a-\frac{i-1}{2}\right) .
\end{aligned}
$$

The multivariate generalization of the beta function is given by

$$
\begin{aligned}
\mathrm{B}_{m}(a, b) & =\int_{0}^{I_{m}} \operatorname{det}(X)^{a-(m+1) / 2} \operatorname{det}\left(I_{m}-X\right)^{b-(m+1) / 2} d X \\
& =\frac{\Gamma_{m}(a) \Gamma_{m}(b)}{\Gamma_{m}(a+b)}=\mathrm{B}_{m}(b, a),
\end{aligned}
$$

where $\operatorname{Re}(a)>(m-1) / 2$ and $\operatorname{Re}(b)>(m-1) / 2$. The generalized hypergeometric function of one matrix is defined by

$$
{ }_{p} F_{q}\left(a_{1}, \ldots, a_{p} ; b_{1}, \ldots, b_{q} ; X\right)=\sum_{k=0}^{\infty} \sum_{\kappa \vdash k} \frac{\left(a_{1}\right)_{\kappa} \cdots\left(a_{p}\right)_{\kappa}}{\left(b_{1}\right)_{\kappa} \cdots\left(b_{q}\right)_{K}} \frac{C_{K}(X)}{k !},
$$


where $a_{i}$ and $b_{j}$ are arbitrary complex numbers when $i=1, \ldots, p$ and $j=1, \ldots, q, X$ is an $m \times m$ complex symmetric matrix, $C_{\kappa}(X)$ is the zonal polynomial of the $m \times m$ complex symmetric matrix $X$ corresponding to the ordered partition $\kappa=\left(k_{1}, \ldots, k_{m}\right)$, with $k_{1} \geq \cdots \geq k_{m} \geq 0$ and $k_{1}+\cdots+k_{m}=k$, and $\sum_{\kappa \vdash k}$ denotes summation over all such partitions $\kappa$. The generalized hypergeometric coefficient $(a)_{\kappa}$ used above is defined by

$$
(a)_{\kappa}=\prod_{i=1}^{m}\left(a-\frac{i-1}{2}\right)_{k_{i}}
$$

where $(a)_{0}=1$ and $(a)_{r}=a(a+1) \cdots(a+r-1)$ for $r=1,2, \ldots$ Conditions for convergence of the series in (2.1) are available in the literature (see, for instance, Constantine [5], James [11]). From (2.1) it follows that

$$
\begin{aligned}
{ }_{0} F_{0}(X) & =\sum_{k=0}^{\infty} \sum_{k \vdash k} \frac{C_{\kappa}(X)}{k !}=\sum_{k=0}^{\infty} \frac{(\operatorname{tr} X)^{k}}{k !}=\operatorname{etr}(X), \\
{ }_{1} F_{0}(a ; X) & =\sum_{k=0}^{\infty} \sum_{k \vdash k} \frac{(a)_{\kappa} C_{K}(X)}{k !}=\operatorname{det}\left(I_{m}-X\right)^{-a}, \\
{ }_{1} F_{1}(a ; c ; X) & =\sum_{k=0}^{\infty} \sum_{k \vdash k} \frac{(a)_{\kappa}}{(c)_{\kappa}} \frac{C_{\kappa}(X)}{k !},
\end{aligned}
$$

and

$$
{ }_{2} F_{1}(a, b ; c ; X)=\sum_{k=0}^{\infty} \sum_{\kappa \vdash k} \frac{(a)_{\kappa}(b)_{\kappa}}{(c)_{\kappa}} \frac{C_{K}(X)}{k !} .
$$

The restriction $\|X\|<1$ is needed to ensure convergence of ${ }_{1} F_{0}(a ; X)$ and ${ }_{2} F_{1}(a ; X)$. The integral representations of the confluent hypergeometric function ${ }_{1} F_{1}$ and the Gauss hypergeometric function ${ }_{2} F_{1}$ are given by

$$
\begin{aligned}
{ }_{1} F_{1}(a ; c ; X)= & \frac{\Gamma_{m}(c)}{\Gamma_{m}(a) \Gamma_{m}(c-a)} \int_{0}^{I_{m}} \operatorname{etr}(R X) \operatorname{det}(R)^{a-(m+1) / 2} \\
& \quad \times \operatorname{det}\left(I_{m}-R\right)^{c-a-(m+1) / 2} d R
\end{aligned}
$$

and for $X<I_{m}$,

$$
\begin{aligned}
{ }_{2} F_{1}(a, b ; c ; X)= & \frac{\Gamma_{m}(c)}{\Gamma_{m}(a) \Gamma_{m}(c-a)} \int_{0}^{I_{m}} \operatorname{det}(R)^{a-(m+1) / 2} \\
& \quad \times \operatorname{det}\left(I_{m}-R\right)^{c-a-(m+1) / 2} \operatorname{det}\left(I_{m}-X R\right)^{-b} d R,
\end{aligned}
$$

where $\operatorname{Re}(a)>(m-1) / 2$ and $\operatorname{Re}(c-a)>(m-1) / 2$. For properties and further results on these functions the reader is referred to Herz [10], Constantine [5], James [11], and Gupta and Nagar [8].

From the above it is easy to see that

$$
{ }_{2} F_{1}\left(a, b ; c ; I_{m}\right)=\frac{\Gamma_{m}(c) \Gamma_{m}(c-a-b)}{\Gamma_{m}(c-a) \Gamma_{m}(c-b)}
$$


and

$$
\begin{aligned}
{ }_{2} F_{1}(a, b ; c ; X) & =\operatorname{det}\left(I_{m}-X\right)^{-b}{ }_{2} F_{1}\left(c-a, b ; c ;-X\left(I_{m}-X\right)^{-1}\right) \\
& =\operatorname{det}\left(I_{m}-X\right)^{c-a-b}{ }_{2} F_{1}(c-a, c-b ; c ; X) .
\end{aligned}
$$

The generalized hypergeometric function with $m \times m$ complex symmetric matrices $X$ and $Y$ is defined by

$$
{ }_{p} F_{q}^{(m)}\left(a_{1}, \ldots, a_{p} ; b_{1}, \ldots, b_{q} ; X, Y\right)=\sum_{k=0}^{\infty} \sum_{\kappa \vdash k} \frac{\left(a_{1}\right)_{\kappa} \cdots\left(a_{p}\right)_{\kappa}}{\left(b_{1}\right)_{\kappa} \cdots\left(b_{q}\right)_{\kappa}} \frac{C_{\kappa}(X) C_{\kappa}(Y)}{C_{\kappa}\left(I_{m}\right) k !} .
$$

It is clear from the above definition that the order of $X$ and $Y$ is unimportant, that is,

$$
{ }_{p} F_{q}^{(m)}\left(a_{1}, \ldots, a_{p} ; b_{1}, \ldots, b_{q} ; X, Y\right)={ }_{p} F_{q}^{(m)}\left(a_{1}, \ldots, a_{p} ; b_{1}, \ldots, b_{q} ; Y, X\right) .
$$

Also, if one of the argument matrices is the identity this function reduces to the oneargument function. Further, the two-matrix argument function ${ }_{p} F_{q}^{(m)}$ can be obtained from the one-matrix function ${ }_{p} F_{q}$ by averaging over the orthogonal group $\mathrm{O}(m)$ using a result given in James [11, Equation (23)], namely,

$$
\int_{\mathrm{O}(m)} C_{K}\left(X H Y H^{T}\right)(d H)=\frac{C_{K}(X) C_{K}(Y)}{C_{K}\left(I_{m}\right)},
$$

where $(d H)$ denotes the normalized invariant measure on $\mathrm{O}(m)$. That is,

$$
\begin{aligned}
{ }_{p} F_{q}^{(m)} & \left(a_{1}, \ldots, a_{p} ; b_{1}, \ldots, b_{q} ; X, Y\right) \\
& =\int_{\mathrm{O}(m)}{ }_{p} F_{q}^{(m)}\left(a_{1}, \ldots, a_{p} ; b_{1}, \ldots, b_{q} ; X H Y H^{T}\right)(d H)
\end{aligned}
$$

(see James [11, equation (30)]). Further, if $\operatorname{Re}(\alpha)>(m-1) / 2$ and $\operatorname{Re}(\beta)>(m-1) / 2$, then

$$
\begin{aligned}
& \int_{0}^{I_{m}} \operatorname{det}(R)^{\alpha-(m+1) / 2} \operatorname{det}\left(I_{m}-R\right)^{\beta-(m+1) / 2}{ }_{p} F_{q}^{(m)}\left(a_{1}, \ldots, a_{p} ; b_{1}, \ldots, b_{q} ; X R, Y\right) d R \\
& \quad=\mathrm{B}_{m}(\alpha, \beta)_{p+1} F_{q+1}^{(m)}\left(a_{1}, \ldots, a_{p}, \alpha ; b_{1}, \ldots, b_{q}, \alpha+\beta ; X, Y\right),
\end{aligned}
$$

which can be obtained by expanding ${ }_{p} F_{q}^{(m)}$ in the integrand in series involving zonal polynomials and integrating term by term using Constantine [5, equation (22)].

Davis [6, 7] introduced a class of polynomials $C_{\phi}^{\kappa, \lambda}(X, Y)$ of $m \times m$ symmetric matrix arguments $X$ and $Y$; these polynomials are invariant under the transformation $(X, Y) \rightarrow\left(H X H^{T}, H Y H^{T}\right)$, where $H \in \mathrm{O}(m)$. For properties and applications of invariant polynomials we refer to Davis [6, 7], Chikuse [4] and Nagar and Gupta [16]. Let $\kappa, \lambda, \phi$ and $\rho$ be ordered partitions of the nonnegative integers $k, \ell, f=k+\ell$ and $r$ 
respectively into not more than $m$ parts. Then

$$
\begin{gathered}
C_{\phi}^{\kappa, \lambda}(X, X)=\theta_{\phi}^{\kappa, \lambda} C_{\phi}(X), \quad \theta_{\phi}^{\kappa, \lambda}=\frac{C_{\phi}^{\kappa, \lambda}\left(I_{m}, I_{m}\right)}{C_{\phi}\left(I_{m}\right)}, \\
C_{\phi}^{\kappa, \lambda}\left(X, I_{m}\right)=\theta_{\phi}^{\kappa, \lambda} \frac{C_{\phi}\left(I_{m}\right) C_{\kappa}(X)}{C_{\kappa}\left(I_{m}\right)}, \\
C_{\kappa}^{\kappa, 0}(X, Y) \equiv C_{\kappa}(X), \quad C_{\lambda}^{0, \lambda}(X, Y) \equiv C_{\lambda}(Y), \\
C_{\kappa}(X) C_{\lambda}(Y)=\sum_{\phi \in \kappa \cdot \lambda} \theta_{\phi}^{\kappa, \lambda} C_{\phi}^{\kappa, \lambda}(X, Y), \quad C_{\kappa}(X) C_{\lambda}(X)=\sum_{\phi \in \kappa \cdot \lambda}\left(\theta_{\phi}^{\kappa, \lambda}\right)^{2} C_{\phi}(X),
\end{gathered}
$$

where $\phi \in \kappa \cdot \lambda$ signifies that the irreducible representation of $\operatorname{Gl}(m, R)$ indexed by $2 \phi$ occurs in the decomposition of the Kronecker product $2 \kappa \otimes 2 \lambda$ of the irreducible representations indexed by $2 \kappa$ and $2 \lambda$. Further, provided that $\operatorname{Re}(\alpha)>(m-1) / 2$ and $\operatorname{Re}(\beta)>(m-1) / 2$,

$$
\begin{gathered}
\int_{0}^{I_{m}} \operatorname{det}(R)^{\alpha-(m+1) / 2} \operatorname{det}\left(I_{m}-R\right)^{\beta-(m+1) / 2} C_{\phi}^{\kappa, \lambda}\left(R, I_{m}-R\right) d R \\
=\frac{\mathrm{B}_{m}(\alpha, \beta)(\alpha)_{\kappa}(\beta)_{\lambda}}{(\alpha+\beta)_{\phi}} \theta_{\phi}^{\kappa, \lambda} C_{\phi}\left(I_{m}\right) .
\end{gathered}
$$

Note that, if $\lambda=0$, then $C_{\kappa}^{\kappa, 0}\left(R, I_{m}-R\right) \equiv C_{\kappa}(R)$ and the above expression reduces to

$$
\int_{0}^{I_{m}} \operatorname{det}(R)^{\alpha-(m+1) / 2} \operatorname{det}\left(I_{m}-R\right)^{\beta-(m+1) / 2} C_{K}(R) d R=\frac{B_{m}(\alpha, \beta)(\alpha)_{K}}{(\alpha+\beta)_{K}} C_{K}\left(I_{m}\right) .
$$

Appell's first hypergeometric function $F_{1}$ and Humbert's confluent hypergeometric function $\boldsymbol{\Phi}_{1}$ of $m \times m$ symmetric matrices $Z_{1}$ and $Z_{2}$ are defined by Saxena et al. [18] and Gupta and Nagar [9]:

$$
\begin{aligned}
& F_{1}\left(a, b_{1}, b_{2} ; c ; Z_{1}, Z_{2}\right) \\
& \quad=\frac{\Gamma_{m}(c)}{\Gamma_{m}(a) \Gamma_{m}(c-a)} \int_{0}^{I_{m}} \frac{\operatorname{det}(V)^{a-(m+1) / 2} \operatorname{det}\left(I_{m}-V\right)^{c-a-(m+1) / 2} d V}{\operatorname{det}\left(I_{m}-V Z_{1}\right)^{b_{1}} \operatorname{det}\left(I_{m}-V Z_{2}\right)^{b_{2}}}
\end{aligned}
$$

and

$$
\begin{aligned}
\boldsymbol{\Phi}_{1}\left[a, b_{1} ; c ; Z_{1}, Z_{2}\right] \\
=\frac{\Gamma_{m}(c)}{\Gamma_{m}(a) \Gamma_{m}(c-a)} \int_{0}^{I_{m}} \frac{\operatorname{det}(V)^{a-(m+1) / 2} \operatorname{det}\left(I_{m}-V\right)^{c-a-(m+1) / 2} d V}{\operatorname{det}\left(I_{m}-V Z_{1}\right)^{b_{1}} \operatorname{etr}\left(-V Z_{2}\right)}
\end{aligned}
$$

respectively, where $\operatorname{Re}(a)>(m-1) / 2$ and $\operatorname{Re}(c-a)>(m-1) / 2$. Note that if $b_{1}=0$, then $F_{1}$ and $\boldsymbol{\Phi}_{1}$ reduce to ${ }_{2} F_{1}$ and ${ }_{1} F_{1}$ respectively. The series expansions for $F_{1}$ and $\boldsymbol{\Phi}_{1}$ are

$$
F_{1}\left(a, b_{1}, b_{2} ; c ; Z_{1}, Z_{2}\right)=\sum_{k=0}^{\infty} \sum_{\ell=0}^{\infty} \sum_{\kappa \vdash k} \sum_{\lambda \vdash \ell} \sum_{\phi \in K \cdot \lambda} \frac{\left(b_{1}\right)_{\kappa}\left(b_{2}\right)_{\lambda}}{k ! \ell !} \frac{(a)_{\phi}}{(c)_{\phi}} C_{\phi}^{\kappa, \lambda}\left(Z_{1}, Z_{2}\right),
$$


where $\left\|Z_{1}\right\|<1,\left\|Z_{2}\right\|<1$, and

$$
\boldsymbol{\Phi}_{1}\left[a, b_{1} ; c ; Z_{1}, Z_{2}\right]=\sum_{k=0}^{\infty} \sum_{\ell=0}^{\infty} \sum_{\kappa \vdash k} \sum_{\lambda \vdash \ell} \sum_{\phi \in \kappa \cdot \lambda} \frac{\left(b_{1}\right)_{\kappa}}{k ! \ell !} \frac{(a)_{\phi}}{(c)_{\phi}} C_{\phi}^{\kappa, \lambda}\left(Z_{1}, Z_{2}\right),
$$

where $\left\|Z_{1}\right\|<1$. Finally, we give the following result known as Hsu's lemma (see Anderson [1, p. 539], Srivastava and Khatri [19, p. 76]).

Lemma 2.1. Suppose that $Y$ is an $m \times n$ matrix of rank $m$ (where $m \leq n$ ) and let $f(Y)$ be a function of $Y$ that depends on $Y Y^{T}$ only, that is, $f(Y)=g\left(Y Y^{T}\right)$ for some $g$. Then, when $W>0$,

$$
\int_{Y Y^{T}=W} f(Y) d Y=\frac{\pi^{n m / 2}}{\Gamma_{m}(n / 2)} \operatorname{det}(W)^{(n-m-1) / 2} g(W) .
$$

\section{The density function}

First we define the matrix-variate Gauss hypergeometric distribution.

Definition 3.1. An $m \times m$ random symmetric positive definite matrix $X$ is said to have a matrix-variate Gauss hypergeometric distribution with parameters $(\alpha, \beta, \gamma, \Xi)$, denoted by $X \sim \mathrm{GH}_{m}(\alpha, \beta, \gamma, \Xi)$, if its p.d.f. is given by

$$
C(\alpha, \beta, \gamma, \Xi) \frac{\operatorname{det}(X)^{\alpha-(m+1) / 2} \operatorname{det}\left(I_{m}-X\right)^{\beta-(m+1) / 2}}{\operatorname{det}\left(I_{m}+\Xi X\right)^{\gamma}},
$$

where $0<X<I_{m}, \quad \alpha>(m-1) / 2, \quad \beta>(m-1) / 2, \quad-\infty<\gamma<\infty, \quad I_{m}+\Xi>0 \quad$ and $C(\alpha, \beta, \gamma, \Xi)$ is the normalizing constant.

The normalizing constant in (3.1) is given by

$$
\begin{aligned}
\{C(\alpha, \beta, \gamma, \Xi)\}^{-1} & =\int_{0}^{I_{m}} \frac{\operatorname{det}(X)^{\alpha-(m+1) / 2} \operatorname{det}\left(I_{m}-X\right)^{\beta-(m+1) / 2}}{\operatorname{det}\left(I_{m}+\Xi X\right)^{\gamma}} d X \\
& =\frac{\Gamma_{m}(\alpha) \Gamma_{m}(\beta)}{\Gamma_{m}(\alpha+\beta)}{ }_{2} F_{1}(\alpha, \gamma ; \alpha+\beta ;-\Xi),
\end{aligned}
$$

where ${ }_{2} F_{1}$ is the Gauss hypergeometric function of matrix argument.

Note that the Gauss hypergeometric function in (3.2) can be expanded in series form using (2.2) if $\|\Xi\|<1$. However, if $\|\Xi\|>I_{m}$, then $\left\|\left(I_{m}+\Xi\right)^{-1} \Xi\right\|<1$ and we use (2.3) to rewrite ${ }_{2} F_{1}(\alpha, \gamma ; \alpha+\beta ;-\Xi)$ in terms of another Gauss hypergeometric function with $\operatorname{argument}\left(I_{m}+\Xi\right)^{-1} \Xi$.

When either $\gamma$ or $\Xi$ is equal to zero, the matrix-variate Gauss hypergeometric density reduces to a matrix-variate beta type 1 density given by

$$
\mathrm{B} 1(X ; m, \alpha, \beta)=\frac{\operatorname{det}(X)^{\alpha-(m+1) / 2} \operatorname{det}\left(I_{m}-X\right)^{\beta-(m+1) / 2}}{\mathrm{~B}_{m}(\alpha, \beta)},
$$


where $0<X<I_{m}$. Further, for $\gamma=\alpha+\beta$ and $\Xi=I_{m}$, the matrix-variate Gauss hypergeometric distribution simplifies to a matrix-variate beta type 3 distribution given by the density

$$
\mathrm{B} 3(X ; m, \alpha, \beta)=\frac{2^{m \alpha} \operatorname{det}(X)^{\alpha-(m+1) / 2} \operatorname{det}\left(I_{m}-X\right)^{\beta-(m+1) / 2}}{\mathrm{~B}_{m}(\alpha, \beta) \operatorname{det}\left(I_{m}+X\right)^{\alpha+\beta}},
$$

where $0<X<I_{m}, \alpha>(m-1) / 2$ and $\beta>(m-1) / 2$. The matrix-variate beta type 1 and beta type 3 distributions have been extensively studied (see, for example, Gupta and Nagar [8,9]). For $\gamma=\alpha+\beta$ and $\Xi=-\left(I_{m}-\Lambda\right)$, where $\Lambda>0$, the matrix-variate Gauss hypergeometric distribution becomes a generalized matrix-variate beta type 1 distribution defined by the density

$$
\mathrm{B} 1(X ; m, \alpha, \beta ; \Lambda)=\frac{\operatorname{det}(\Lambda)^{\alpha} \operatorname{det}(X)^{\alpha-(m+1) / 2} \operatorname{det}\left(I_{m}-X\right)^{\beta-(m+1) / 2}}{\mathrm{~B}_{m}(\alpha, \beta) \operatorname{det}\left(I_{m}-\left(I_{m}-\Lambda\right) X\right)^{\alpha+\beta}},
$$

where $0<X<I_{m}, \alpha>(m-1) / 2$ and $\beta>(m-1) / 2$.

When $m=1$, the matrix-variate Gauss hypergeometric distribution reduces to a univariate Gauss hypergeometric distribution.

The cumulative distribution function of $X$ is obtained as

$$
\begin{aligned}
P(X<\Omega)= & C(\alpha, \beta, \gamma, \Xi) \int_{0}^{\Omega} \frac{\operatorname{det}(X)^{\alpha-(m+1) / 2} \operatorname{det}\left(I_{m}-X\right)^{\beta-(m+1) / 2}}{\operatorname{det}\left(I_{m}+\Xi X\right)^{\gamma}} d X \\
= & C(\alpha, \beta, \gamma, \Xi) \operatorname{det}(\Omega)^{\alpha} \\
& \quad \times \int_{0}^{I_{m}} \frac{\operatorname{det}(W)^{\alpha-(m+1) / 2} \operatorname{det}\left(I_{m}-W\right)^{\alpha+(m+1) / 2-\alpha-(m+1) / 2}}{\operatorname{det}\left(I_{m}-\Omega W\right)^{(m+1) / 2-\beta} \operatorname{det}\left(I_{m}+\Omega^{1 / 2} \Xi \Omega^{1 / 2} W\right)^{\gamma}} d W,
\end{aligned}
$$

where the last line has been obtained by substituting $W=\Omega^{-1 / 2} X \Omega^{-1 / 2}$ with the Jacobian $J(X \rightarrow W)=\operatorname{det}(\Omega)^{(m+1) / 2}$. Now, applying (2.10), we get

$$
\begin{aligned}
P(X<\Omega)=C & (\alpha, \beta, \gamma, \Xi) \operatorname{det}(\Omega)^{\alpha} \mathrm{B}_{m}\left(\alpha, \frac{m+1}{2}\right) \\
& \times F_{1}\left(\alpha, \frac{m+1}{2}-\beta, \gamma ; \alpha+\frac{m+1}{2} ; \Omega,-\Omega^{1 / 2} \Xi \Omega^{1 / 2}\right) .
\end{aligned}
$$

The moment generating function $M_{X}(Z)$ of $X$ is derived as

$$
M_{X}(Z)=C(\alpha, \beta, \gamma, \Xi) \int_{0}^{I_{m}} \operatorname{etr}(Z X) \frac{\operatorname{det}(X)^{\alpha-(m+1) / 2} \operatorname{det}\left(I_{m}-X\right)^{\beta-(m+1) / 2}}{\operatorname{det}\left(I_{m}+\Xi X\right)^{\gamma}} d X,
$$

where $Z$ is the $m \times m$ matrix $\left(\left(1+\delta_{i j}\right) z_{i j} / 2\right)$. Now, using the integral representation (2.11),

$$
\begin{aligned}
M_{X}(Z) & =C(\alpha, \beta, \gamma, \Xi) \mathrm{B}_{m}(\alpha, \beta) \boldsymbol{\Phi}_{1}[\alpha, \gamma ; \alpha+\beta ;-\Xi, Z] \\
& =\frac{\boldsymbol{\Phi}_{1}[\alpha, \gamma ; \alpha+\beta ;-\Xi, Z]}{{ }_{2} F_{1}(\alpha, \gamma ; \alpha+\beta ;-\Xi)} .
\end{aligned}
$$




\section{Properties}

In this section we study some properties of Gauss hypergeometric distributed random matrices.

Theorem 4.1. Suppose that $X \sim \mathrm{GH}_{m}(\alpha, \beta, \gamma, \Xi)$. Then

$$
I_{m}-X \sim \mathrm{GH}_{m}\left(\beta, \alpha, \gamma,-\left(I_{m}+\Xi\right)^{-1} \Xi\right) .
$$

Theorem 4.2. Suppose that $X \sim \mathrm{GH}_{m}(\alpha, \beta, \gamma, \Xi)$ and let $A$ be an $m \times m$ constant nonsingular matrix. Then the density of $Y=A X A^{T}$ is given by

$$
C(\alpha, \beta, \gamma, \Xi) \frac{\operatorname{det}(Y)^{\alpha-(m+1) / 2} \operatorname{det}\left(A A^{T}-Y\right)^{\beta-(m+1) / 2}}{\operatorname{det}\left(A A^{T}\right)^{\alpha+\beta-(m+1) / 2} \operatorname{det}\left(I_{m}+\left(A^{T}\right)^{-1} \Xi A^{-1} Y\right)^{\gamma}},
$$

where $0<Y<A A^{T}$.

Proof. In the p.d.f. (3.1) of $X$, by making the transformation $Y=A X A^{T}$ with the Jacobian $J(X \rightarrow Y)=\operatorname{det}(A)^{-(m+1)}$, the density of $Y$ is obtained.

We will write $Y \sim \mathrm{GH}_{m}(\alpha, \beta, \gamma, \Xi, A)$. In the next theorem, it is shown that the matrix-variate Gauss hypergeometric distribution is orthogonally invariant.

Theorem 4.3. Suppose that $X \sim \mathrm{GH}_{m}\left(\alpha, \beta, \gamma, I_{m}\right)$ and $H$ is an orthogonal $m \times$ $m$ matrix whose elements are either constants or random variables distributed independently of $X$. Then the distribution of $X$ is invariant under the transformation $X \rightarrow H X H^{T}$.

Proof. First, let $H$ be a constant orthogonal matrix. Then, from Theorem 4.2, $H X H^{T} \sim \mathrm{GH}_{m}\left(\alpha, \beta, \gamma, I_{m}\right)$ since $H H^{T}=I_{m}$. If, however, $H$ is a random orthogonal matrix, then the conditional distribution of $H X H^{T} \mid H \sim \mathrm{GH}_{m}\left(\alpha, \beta, \gamma, I_{m}\right)$. Since this distribution does not depend on $H$, we have $H X H^{T} \sim \mathrm{GH}_{m}\left(\alpha, \beta, \gamma, I_{m}\right)$.

The relationship between matrix-variate beta type 1, matrix-variate type 2 and matrix-variate Gauss hypergeometric distributions is now analyzed. First, we give the definition of the matrix-variate beta type 2 distribution.

Definition 4.4. An $m \times m$ random symmetric positive definite matrix $V$ is said to have a matrix-variate beta type 2 distribution with parameters $(\alpha, \beta)$, denoted by $V \sim \mathrm{B} 2(m, \alpha, \beta)$, if its p.d.f. is given by

$$
\frac{\operatorname{det}(V)^{\alpha-(m+1) / 2} \operatorname{det}\left(I_{m}+V\right)^{-(\alpha+\beta)}}{\mathrm{B}_{m}(\alpha, \beta)},
$$

where $V>0, \alpha>(m-1) / 2, \beta>(m-1) / 2$, and $B_{m}(\alpha, \beta)$ is the multivariate beta function.

The density (4.1) can be obtained from (3.3) by transforming $X=\left(I_{m}+V\right)^{-1} V$, together with the Jacobian $J(X \rightarrow V)=\operatorname{det}\left(I_{m}+V\right)^{-(m+1)}$. 
Theorem 4.5. Suppose that $U \sim \mathrm{B} 1(m, \alpha, \beta)$. Then

$$
\left(I_{m}+U\right)^{-1}\left(I_{m}-U\right) \sim \mathrm{GH}_{m}\left(\beta, \alpha, \alpha+\beta, I_{m}\right)
$$

and

$$
\left(2 I_{m}-U\right)^{-1} U \sim \mathrm{GH}_{m}\left(\alpha, \beta, \alpha+\beta, I_{m}\right) .
$$

Theorem 4.6. Suppose that $V \sim \mathrm{B} 2(m, \alpha, \beta)$. Then

$$
\left(I_{m}+2 V\right)^{-1} \sim \mathrm{GH}_{m}\left(\beta, \alpha, \alpha+\beta, I_{m}\right)
$$

and

$$
\left(2 I_{m}+V\right)^{-1} V \sim \mathrm{GH}_{m}\left(\alpha, \beta, \alpha+\beta, I_{m}\right) .
$$

Theorem 4.7. Suppose that $X \sim \mathrm{GH}_{m}(\alpha, \beta, \gamma, \Xi)$ and $Y=\left(I_{m}+X\right)^{-1}\left(I_{m}-X\right)$. Then the density of $Y$, for $0<Y<I_{m}$, is given by

$$
C(\alpha, \beta, \gamma, \Xi) \frac{2^{m \beta} \operatorname{det}(Y)^{\beta-(m+1) / 2} \operatorname{det}\left(I_{m}-Y\right)^{\alpha-(m+1) / 2}}{\operatorname{det}\left(I_{m}+\Xi\right)^{\gamma} \operatorname{det}\left(I_{m}+Y\right)^{\alpha+\beta-\gamma} \operatorname{det}\left(I_{m}+\left(I_{m}+\Xi\right)^{-1}\left(I_{m}-\Xi\right) Y\right)^{\gamma}} .
$$

Proof. Since $Y=\left(I_{m}+X\right)^{-1}\left(I_{m}-X\right)$, the Jacobian $J(X \rightarrow Y)$ of the transformation is $2^{m(m+1) / 2} \operatorname{det}\left(I_{m}+Y\right)^{-(m+1)}$. Now making these substitutions in the density of $X$, the result follows.

Corollary 4.8. Suppose that $X \sim \mathrm{GH}_{m}\left(\alpha, \beta, \gamma, I_{m}\right)$. Then

$$
\left.\left(I_{m}+X\right)^{-1}\left(I_{m}-X\right) \sim \mathrm{GH}_{m}\left(\beta, \alpha, \alpha+\beta-\gamma, I_{m}\right)\right) .
$$

Corollary 4.9. Suppose that $X \sim \mathrm{GH}_{m}(\alpha, \beta, \alpha+\beta, \Xi)$. Then

$$
\left(I_{m}+X\right)^{-1}\left(I_{m}-X\right) \sim \mathrm{GH}_{m}\left(\beta, \alpha, \alpha+\beta,\left(I_{m}+\Xi\right)^{-1}\left(I_{m}-\Xi\right)\right) .
$$

Theorem 4.10. Suppose that $X \sim \mathrm{GH}_{m}(\alpha, \beta, \gamma, \Xi)$ and $Y=2\left(I_{m}+X\right)^{-1} X$. Then the density of $Y$, for $0<Y<I_{m}$, is given by

$$
C(\alpha, \beta, \gamma, \Xi) \frac{2^{m \beta} \operatorname{det}(Y)^{\alpha-(m+1) / 2} \operatorname{det}\left(I_{m}-Y\right)^{\beta-(m+1) / 2}}{\operatorname{det}\left(2 I_{m}-Y\right)^{\alpha+\beta-\gamma} \operatorname{det}\left(2 I_{m}-\left(I_{m}-\Xi\right) Y\right)^{\gamma}} .
$$

Proof. Since $Y=2\left(I_{m}+X\right)^{-1} X$, the Jacobian $J(X \rightarrow Y)$ of the transformation is $2^{m(m+1) / 2} \operatorname{det}\left(2 I_{m}-Y\right)^{-(m+1)}$. Now, making these substitutions in the density of $X$, the result follows.

Corollary 4.11. Suppose that $X \sim \mathrm{GH}_{m}(\alpha, \beta, \alpha+\beta, \Xi)$. Then

$$
2\left(I_{m}+X\right)^{-1} X \sim \mathrm{GH}_{m}\left(\alpha, \beta, \alpha+\beta,-\left(I_{m}-\Xi\right) / 2\right) .
$$

Corollary 4.12. Suppose that $X \sim \mathrm{GH}_{m}\left(\alpha, \beta, \gamma, I_{m}\right)$. Then

$$
2\left(I_{m}+X\right)^{-1} X \sim \mathrm{GH}_{m}\left(\alpha, \beta, \alpha+\beta-\gamma,-I_{m} / 2\right) .
$$


Let $V \sim \mathrm{B} 2(m, a, b)$ and $V=\left(\begin{array}{ll}V_{11} & V_{12} \\ V_{21} & V_{22}\end{array}\right)$, where $V_{11}$ is a $q \times q$ matrix and $V_{22}$ is an $(m-q) \times(m-q)$ matrix, and let

$$
V_{11 \cdot 2}=V_{11}-V_{12} V_{22}^{-1} V_{21}
$$

It is well known that $V_{11.2}$ and $V_{22}$ are distributed independently (Gupta and Nagar [8]), and that $V_{11 \cdot 2} \sim \mathrm{B} 2(q, a-(m-q) / 2, b)$ and $V_{22} \sim \mathrm{B} 2(m-q, a, b-q / 2)$. By Theorem 4.6, if $V \sim \mathrm{B} 2(m, a, b)$, then $X=\left(I_{m}+2 V\right)^{-1} \sim \mathrm{GH}_{m}\left(b, a, a+b, I_{m}\right)$. Furthermore,

$$
X^{-1}=\left(\begin{array}{ll}
X^{11} & X^{12} \\
X^{21} & X^{22}
\end{array}\right)=\left(\begin{array}{cc}
I_{q}+2 V_{11} & 2 V_{12} \\
2 V_{21} & I_{m-q}+2 V_{22}
\end{array}\right) .
$$

That is, $X^{22}=X_{22 \cdot 1}^{-1}=I_{m-q}+2 V_{22}$ and $X_{22 \cdot 1}=\left(I_{m-q}+2 V_{22}\right)^{-1}$. Now, since $V_{22} \sim$ $\mathrm{B} 2(m-q, a, b-q / 2)$,

$$
X_{22 \cdot 1}=\left(I_{m-q}+2 V_{22}\right)^{-1} \sim \mathrm{GH}_{m-q}\left(b-q / 2, a, a+b-q / 2, I_{m-q}\right) .
$$

Thus, we have the following result.

Theorem 4.13. If $X \sim \mathrm{GH}_{m}\left(\alpha, \beta, \alpha+\beta, I_{m}\right)$, then

$$
X_{22 \cdot 1} \sim \mathrm{GH}_{m-q}\left(\alpha-q / 2, \beta, \alpha+\beta-q / 2, I_{m-q}\right) .
$$

Next, we derive the joint density of $X_{11}$ and $X_{22 \cdot 1}$ for $X \sim \mathrm{GH}_{m}\left(\alpha, \beta, \gamma, I_{m}\right)$.

Theorem 4.14. Suppose that $X=\left(\begin{array}{ll}X_{11} & X_{12} \\ X_{21} & X_{22}\end{array}\right)$, where $X_{11}$ is a $q \times q$ matrix, and define $X_{22 \cdot 1}=X_{22}-X_{21} X_{11}^{-1} X_{12}$. If $X \sim \mathrm{GH}_{m}\left(\alpha, \beta, \gamma, I_{m}\right)$, then the joint density of $X_{11}$ and $X_{22 \cdot 1}$ is given by

$$
\begin{aligned}
C\left(\alpha, \beta, \gamma, I_{m}\right) \frac{\pi^{q(m-q) / 2} \Gamma_{m-q}(\beta-q / 2)}{\Gamma_{m-q}(\beta)} \frac{\operatorname{det}\left(X_{11}\right)^{\alpha-(q+1) / 2} \operatorname{det}\left(I_{q}-X_{11}\right)^{\beta-(q+1) / 2}}{\operatorname{det}\left(I_{q}+X_{11}\right)^{\gamma}} \\
\quad \times \frac{\operatorname{det}\left(X_{22 \cdot 1}\right)^{\alpha-(m+1) / 2} \operatorname{det}\left(I_{m-q}-X_{22 \cdot 1}\right)^{\beta-(m-q+1) / 2}}{\operatorname{det}\left(I_{m-q}+X_{22 \cdot 1}\right)^{\gamma}} F_{1}^{(\delta)}\left(\frac{1}{2} \delta, \gamma ; \beta ;-A, B\right),
\end{aligned}
$$

where $0<X_{11}<I_{m}, 0<X_{22 \cdot 1}<I_{m}, A=\left(I_{m-q}-X_{22 \cdot 1}\right)^{1 / 2}\left(I_{m-q}+X_{22 \cdot 1}\right)^{-1}\left(I_{m-q}-X_{22 \cdot 1}\right)^{1 / 2}$, $B=\left(I_{q}-X_{11}\right)^{1 / 2}\left(I_{q}+X_{11}\right)^{-1}\left(I_{q}-X_{11}\right)^{1 / 2}$ and $\delta=\max \{q, m-q\}$.

Proof. From the partition of $X$,

$$
\begin{gathered}
\operatorname{det}(X)=\operatorname{det}\left(X_{11}\right) \operatorname{det}\left(X_{22 \cdot 1}\right), \\
\operatorname{det}\left(I_{m}-X\right)=\operatorname{det}\left(I_{q}-X_{11}\right) \operatorname{det}\left(I_{m-q}-X_{22 \cdot 1}-X_{21} X_{11}^{-1}\left(I_{q}-X_{11}\right)^{-1} X_{12}\right), \\
\operatorname{det}\left(I_{m}+X\right)=\operatorname{det}\left(I_{q}+X_{11}\right) \operatorname{det}\left(I_{m-q}+X_{22 \cdot 1}+X_{21} X_{11}^{-1}\left(I_{q}+X_{11}\right)^{-1} X_{12}\right) .
\end{gathered}
$$

Substituting (4.3), (4.4) and (4.5) in the density of $X$ and making the transformation

$$
\begin{aligned}
X_{11} & =X_{11}, \\
Y & =\left(I_{m-q}-X_{22 \cdot 1}\right)^{-1 / 2} X_{21} X_{11}^{-1 / 2}\left(I_{q}-X_{11}\right)^{-1 / 2}, \\
X_{22 \cdot 1} & =X_{22}-X_{21} X_{11}^{-1} X_{12},
\end{aligned}
$$


whose Jacobian $J\left(X_{11}, X_{22}, X_{21} \rightarrow X_{11}, X_{22 \cdot 1}, Y\right)$ is equal to

$$
\operatorname{det}\left(I_{m-q}-X_{22 \cdot 1}\right)^{q / 2} \operatorname{det}\left(I_{q}-X_{11}\right)^{(m-q) / 2} \operatorname{det}\left(X_{11}\right)^{(m-q) / 2},
$$

and integrating $Y$, we get the joint density of $X_{11}$ and $X_{22 \cdot 1}$ as

$$
\begin{aligned}
& C\left(\alpha, \beta, \gamma, I_{m}\right) \frac{\operatorname{det}\left(X_{11}\right)^{\alpha-(q+1) / 2} \operatorname{det}\left(I_{q}-X_{11}\right)^{\beta-(q+1) / 2}}{\operatorname{det}\left(I_{q}+X_{11}\right)^{\gamma}} \\
& \quad \times \frac{\operatorname{det}\left(X_{22 \cdot 1}\right)^{\alpha-(m+1) / 2} \operatorname{det}\left(I_{m-q}-X_{22 \cdot 1}\right)^{\beta-(m-q+1) / 2}}{\operatorname{det}\left(I_{m-q}+X_{22 \cdot 1}\right)^{\gamma}} g(A, B),
\end{aligned}
$$

where when $m-q \leq q$,

$$
\begin{aligned}
g(A, B) & =\int_{I_{m-q}-Y Y^{T}>0} \operatorname{det}\left(I_{m-q}-Y Y^{T}\right)^{\beta-(m+1) / 2} \operatorname{det}\left(I_{m-q}+A Y B Y^{T}\right)^{-\gamma} d Y \\
& =\int_{0}^{I_{m-q}} \int_{Y Y^{T}=Z} \operatorname{det}\left(I_{m-q}-Y Y^{T}\right)^{\beta-(m+1) / 2}{ }_{1} F_{0}^{(q)}\left(\gamma ;-Y^{T} A Y B\right) d Y d Z,
\end{aligned}
$$

and when $m-q>q$,

$$
g(A, B)=\int_{0}^{I_{q}} \int_{Y^{T} Y=Z} \operatorname{det}\left(I_{q}-Y^{T} Y\right)^{\beta-(m+1) / 2}{ }_{1} F_{0}^{(m-q)}\left(\gamma ;-A Y B Y^{T}\right) d Y d Z
$$

We evaluate $g(A, B)$ when $m-q \leq q$ given in (4.7). Since $g(A, B)=g\left(A, H^{T} B H\right)$, when $H \in \mathrm{O}(q)$, integrating $H$ in $g\left(A, H^{T} B H\right)$ using (2.5),

$$
\begin{aligned}
g(A, B) & =\int_{0}^{I_{m-q}} \int_{Y Y^{T}=Z} \operatorname{det}\left(I_{m-q}-Y Y^{T}\right)^{\beta-(m+1) / 2}{ }_{1} F_{0}^{(q)}\left(\gamma ;-A Y Y^{T}, B\right) d Y d Z \\
& =\frac{\pi^{q(m-q) / 2}}{\Gamma_{m-q}(q / 2)} \int_{0}^{I_{m-q}} \operatorname{det}(Z)^{(q-m+q-1) / 2} \operatorname{det}\left(I_{m-q}-Z\right)^{\beta-(m+1) / 2}{ }_{1} F_{0}^{(q)}(\gamma ;-A Z, B) d Z \\
& =\frac{\pi^{q(m-q) / 2} \Gamma_{m-q}(\beta-q / 2)}{\Gamma_{m-q}(\beta)}{ }_{2} F_{1}^{(q)}\left(\frac{1}{2} q, \gamma ; \beta ;-A, B\right),
\end{aligned}
$$

where the last two lines have been obtained by using Lemma 2.1 and (2.6).

Substituting $g(A, B)$ in (4.6), we get the joint density of $X_{11}$ and $X_{22 \cdot 1}$ as

$$
\begin{aligned}
C\left(\alpha, \beta, \gamma, I_{m}\right) \frac{\pi^{q(m-q) / 2} \Gamma_{m-q}(\beta-q / 2)}{\Gamma_{m-q}(\beta)} \frac{\operatorname{det}\left(X_{11}\right)^{\alpha-(q+1) / 2} \operatorname{det}\left(I_{q}-X_{11}\right)^{\beta-(q+1) / 2}}{\operatorname{det}\left(I_{q}+X_{11}\right)^{\gamma}} \\
\quad \times \frac{\operatorname{det}\left(X_{22 \cdot 1}\right)^{\alpha-(m+1) / 2} \operatorname{det}\left(I_{m-q}-X_{22 \cdot 1}\right)^{\beta-(m-q+1) / 2}}{\operatorname{det}\left(I_{m-q}+X_{22 \cdot 1}\right)^{\gamma}}{ }_{2}^{(q)}\left(\frac{1}{2} q, \gamma ; \beta ;-A, B\right) .
\end{aligned}
$$


When $q<m-q$, using (4.8) and following similar steps, we get

$$
\begin{aligned}
& g(A, B)= \int_{0}^{I_{q}} \int_{Y^{T} Y=Z} \operatorname{det}\left(I_{q}-Y^{T} Y\right)^{\beta-(m+1) / 2}{ }_{1} F_{0}^{(m-q)}\left(\gamma ;-A, B Y^{T} Y\right) d Y d Z \\
&= \frac{\pi^{q(m-q) / 2}}{\Gamma_{q}[(m-q) / 2]} \int_{0}^{I_{q}} \operatorname{det}(Z)^{(m-q-q-1) / 2} \operatorname{det}\left(I_{m-q}-Z\right)^{\beta-(m+1) / 2} \\
& \quad \times{ }_{1} F_{0}^{(m-q)}(\gamma ;-A, B Z) d Z \\
&=\frac{\pi^{q(m-q) / 2} \Gamma_{q}[\beta-(m-q) / 2]}{\Gamma_{q}(\beta)}{ }_{2} F_{1}^{(m-q)}\left(\frac{1}{2}(m-q), \gamma ; \beta ;-A, B\right)
\end{aligned}
$$

and the joint density of $X_{11}$ and $X_{22 \cdot 1}$ is given by

$$
\begin{aligned}
C\left(\alpha, \beta, \gamma, I_{m}\right) & \frac{\pi^{q(m-q) / 2} \Gamma_{q}[\beta-(m-q) / 2]}{\Gamma_{q}(\beta)} \frac{\operatorname{det}\left(X_{11}\right)^{\alpha-(q+1) / 2} \operatorname{det}\left(I_{q}-X_{11}\right)^{\beta-(q+1) / 2}}{\operatorname{det}\left(I_{q}+X_{11}\right)^{\gamma}} \\
& \times \frac{\operatorname{det}\left(X_{22 \cdot 1}\right)^{\alpha-(m+1) / 2} \operatorname{det}\left(I_{m-q}-X_{22 \cdot 1}\right)^{\beta-(m-q+1) / 2}}{\operatorname{det}\left(I_{m-q}+X_{22 \cdot 1}\right)^{\gamma}}{ }_{2} F_{1}^{(m-q)}\left(\frac{1}{2}(m-q), \gamma ; \beta ;-A, B\right) .
\end{aligned}
$$

Now, by noting that

$$
\frac{\Gamma_{m-q}(\beta-q / 2)}{\Gamma_{m-q}(\beta)}=\frac{\Gamma_{q}[\beta-(m-q) / 2]}{\Gamma_{q}(\beta)}
$$

the desired result is obtained.

By substituting $\gamma=0$ in the above theorem, it is easy to see that if $X \sim \mathrm{B} 1(m, \alpha, \beta)$, then $X_{11} \sim \mathrm{B} 1(q, \alpha, \beta)$ and $X_{22 \cdot 1} \sim \mathrm{B} 1(m-q, \alpha-q / 2, \beta)$; moreover, $X_{11}$ and $X_{22 \cdot 1}$ are independent. Further, substituting $\gamma=\alpha+\beta$ in the joint density of $X_{11}$ and $X_{22 \cdot 1}$ and integrating $X_{11}$ using

$$
\begin{aligned}
2^{q \alpha} & \int_{0}^{I_{q}} \frac{\operatorname{det}\left(X_{11}\right)^{\alpha-(q+1) / 2} \operatorname{det}\left(I_{q}-X_{11}\right)^{\beta-(q+1) / 2}}{\operatorname{det}\left(I_{q}+X_{11}\right)^{\alpha+\beta}}{ }_{2} F_{1}^{(q)}\left(\frac{1}{2} q, \alpha+\beta ; \beta ;-A, B\right) d X_{11} \\
& =\int_{0}^{I_{q}} \operatorname{det}(B)^{\beta-(q+1) / 2} \operatorname{det}\left(I_{q}-B\right)^{\alpha-(q+1) / 2}{ }_{2} F_{1}^{(q)}\left(\frac{1}{2} q, \alpha+\beta ; \beta ;-A, B\right) d B \\
& =\mathrm{B}_{q}(\alpha, \beta){ }_{3} F_{2}^{(q)}\left(\beta, \frac{1}{2} q, \alpha+\beta ; \beta, \alpha+\beta ;-A\right) \\
& =\mathrm{B}_{q}(\alpha, \beta) \operatorname{det}\left(I_{m-q}+A\right)^{-q / 2}=2^{-(m-q) q / 2} \mathrm{~B}_{q}(\alpha, \beta) \operatorname{det}\left(I_{m-q}+X_{22 \cdot 1}\right)^{q / 2}
\end{aligned}
$$

we see that $X_{22 \cdot 1} \sim \mathrm{GH}_{m-q}\left(\alpha-q / 2, \beta, \alpha+\beta-q / 2, I_{m-q}\right)$ if $X \sim \mathrm{GH}_{m}\left(\alpha, \beta, \alpha+\beta, I_{m}\right)$, as in Theorem 4.13.

Substituting $m=2$ and $q=1$ in (4.2), the joint density of $X_{11} \equiv x_{11}$ and $X_{22 \cdot 1} \equiv x_{22 \cdot 1}$ simplifies to

$$
\begin{gathered}
\frac{\Gamma(\alpha+\beta) \Gamma(\alpha+\beta-1 / 2)}{\Gamma(\alpha) \Gamma(\alpha-1 / 2) \Gamma^{2}(\beta)_{2} F_{1}\left(\alpha, \gamma ; \alpha+\beta ;-I_{2}\right)} \frac{x_{11}^{\alpha-1}\left(1-x_{11}\right)^{\beta-1}}{\left(1+x_{11}\right)^{\gamma}} \\
\times \frac{x_{22 \cdot 1}^{\alpha-3 / 2}\left(1-x_{22 \cdot 1}\right)^{\beta-1}}{\left(1+x_{22 \cdot 1}\right)^{\gamma}} \sum_{j=0}^{\infty} \frac{(1 / 2)_{j}(\gamma)_{j}}{(\beta)_{j} j !}\left(-\frac{1-x_{11}}{1+x_{11}}\right)^{j}\left(\frac{1-x_{22 \cdot 1}}{1+x_{22} \cdot 1}\right)^{j},
\end{gathered}
$$


where $0<x_{11}<1$ and $0<x_{22.1}<1$. Further, integrating (4.9) with respect to $x_{11}$ and $x_{22.1}$ and simplifying the resulting expression, we get

$$
\begin{aligned}
{ }_{2} F_{1}\left(\alpha, \gamma ; \alpha+\beta ;-I_{2}\right)=\sum_{j=0}^{\infty} & \frac{(-1)^{j}(1 / 2)_{j}(\gamma)_{j}(\beta)_{j}}{(\alpha+\beta)_{j}(\alpha+\beta-1 / 2)_{j} j !}{ }_{2} F_{1}(\alpha, \gamma+j ; \alpha+\beta+j ;-1) \\
& \times{ }_{2} F_{1}\left(\alpha-\frac{1}{2}, \gamma+j ; \alpha+\beta-\frac{1}{2}+j ;-1\right) .
\end{aligned}
$$

Using (2.3), the above identity can also be written as

$$
\begin{aligned}
{ }_{2} F_{1}\left(\beta, \gamma ; \alpha+\beta ; \frac{I_{2}}{2}\right)=\sum_{j=0}^{\infty} & \frac{(-1)^{j}(1 / 2)_{j}(\gamma)_{j}(\beta)_{j}}{2^{2 j}(\alpha+\beta)_{j}(\alpha+\beta-1 / 2)_{j} j !}{ }_{2} F_{1}\left(\beta+j, \gamma+j ; \alpha+\beta+j ; \frac{1}{2}\right) \\
& \times{ }_{2} F_{1}\left(\beta+j, \gamma+j ; \alpha+\beta-\frac{1}{2}+j ; \frac{1}{2}\right)
\end{aligned}
$$

which for $\gamma=\alpha+\beta$ gives an interesting summation formula,

$$
2^{\beta}=\sum_{j=0}^{\infty} \frac{(-1)^{j}(1 / 2)_{j}(\beta)_{j}}{2^{j}(\alpha+\beta-1 / 2)_{j} j !}{ }_{2} F_{1}\left(\beta+j, \alpha+\beta+j ; \alpha+\beta-\frac{1}{2}+j ; \frac{1}{2}\right) .
$$

The distribution of $\left(A X^{-1} A^{T}\right)^{-1}$, where $A$ is a $q \times m$ constant matrix of rank $q$ (where $q \leq m)$, is now derived.

THeOREM 4.15. Suppose that $A$ is a $q \times m$ constant matrix of rank $q$, where $q \leq m$. If $X \sim \mathrm{GH}_{m}\left(\alpha, \beta, \alpha+\beta, I_{m}\right)$, then

$$
\left(A A^{T}\right)^{1 / 2}\left(A X^{-1} A^{T}\right)^{-1}\left(A A^{T}\right)^{1 / 2} \sim \mathrm{GH}_{q}\left(\alpha-\frac{m-q}{2}, \beta, \alpha+\beta-\frac{m-q}{2}, I_{q}\right) .
$$

Proof. Write $A=M\left(I_{q}, 0\right) G$, where $M$ is a nonsingular $q \times q$ matrix and $G$ is an orthogonal $m \times m$ matrix. Now,

$$
\begin{aligned}
& \left.\left(A X^{-1} A^{T}\right)^{-1}=\left(\begin{array}{ll}
M\left(I_{q}\right. & 0
\end{array}\right) G X^{-1} G^{T}\left(\begin{array}{ll}
I_{q} & 0
\end{array}\right)^{T} M^{T}\right)^{-1} \\
& =\left(M^{T}\right)^{-1}\left[\left(\begin{array}{ll}
I_{q} & 0
\end{array}\right) Y^{-1}\left(\begin{array}{c}
I_{q} \\
0
\end{array}\right)\right]^{-1} M^{-1} \\
& =\left(M^{T}\right)^{-1}\left(Y^{11}\right)^{-1} M^{-1} \text {, }
\end{aligned}
$$

where

$$
Y=\left(\begin{array}{ll}
Y_{11} & Y_{12} \\
Y_{21} & Y_{22}
\end{array}\right)=G X G^{T} \sim \mathrm{GH}_{m}\left(\alpha, \beta, \alpha+\beta, I_{m}\right),
$$

$Y_{11}$ is a $q \times q$ matrix and $Y^{11}=\left(Y_{11}-Y_{12} Y_{22}^{-1} Y_{21}\right)^{-1}=Y_{11 \cdot 2}^{-1}$. From Theorem 4.13,

$$
Y_{11.2} \sim \mathrm{GH}_{q}\left(\alpha-(m-q) / 2, \beta, \alpha+\beta-(m-q) / 2, I_{q}\right),
$$


and from Theorem 4.2,

$$
Z=\left(M^{T}\right)^{-1} Y_{11 \cdot 2} M^{-1} \sim \mathrm{GH}_{q}\left(\alpha-(m-q) / 2, \beta, \alpha+\beta-(m-q) / 2, I_{q},\left(M^{T}\right)^{-1}\right),
$$

with the p.d.f. proportional to

$$
\frac{\operatorname{det}(Z)^{\alpha-(m+1) / 2} \operatorname{det}\left(\left(M M^{T}\right)^{-1}-Z\right)^{\beta-(q+1) / 2}}{\operatorname{det}\left(M M^{T}\right)^{-(\alpha+\beta)+(m+1) / 2} \operatorname{det}\left(I_{q}+M M^{T} Z\right)^{\alpha+\beta-(m-q) / 2}},
$$

where $0<Z<\left(M M^{T}\right)^{-1}$. Now, noting that $M M^{T}=A A^{T}$ and making the transformation $S=\left(A A^{T}\right)^{1 / 2} Z\left(A A^{T}\right)^{1 / 2}$ with the Jacobian $J(Z \rightarrow S)=\operatorname{det}\left(A A^{T}\right)^{-(m+1) / 2}$ in the above density, we get the desired result.

Corollary 4.16. Suppose that $X \sim \mathrm{GH}_{m}\left(\alpha, \beta, \alpha+\beta, I_{m}\right)$ and $\mathbf{a} \in \mathbb{R}^{m} \backslash\{\mathbf{0}\}$. Then

$$
\mathbf{a}^{T} \mathbf{a}\left(\mathbf{a}^{T} X^{-1} \mathbf{a}\right)^{-1} \sim \mathrm{GH}(\alpha-(m-1) / 2, \beta, \alpha+\beta-(m-1) / 2,1) .
$$

In the above corollary, the distribution of $\mathbf{a}^{T} \mathbf{a}\left(\mathbf{a}^{T} X^{-1} \mathbf{a}\right)^{-1}$ does not depend on $\mathbf{a}$. Thus, if $\mathbf{y}$ is a random vector in $\mathbb{R}^{m}$, independent of $X$, and $P(\mathbf{y} \neq \mathbf{0})=1$, then it follows that $\mathbf{y}^{T} \mathbf{y}\left(\mathbf{y}^{T} X^{-1} \mathbf{y}\right)^{-1} \sim \mathrm{GH}(\alpha-(m-1) / 2, \beta, \alpha+\beta-(m-1) / 2,1)$.

Theorem 4.17. Suppose that $X \sim \mathrm{GH}_{m}(\alpha, \beta, \gamma, \Xi)$. Then

$$
\begin{aligned}
E\left[\frac{\operatorname{det}(X)^{r} \operatorname{det}\left(I_{m}-X\right)^{s}}{\operatorname{det}\left(I_{m}+\Xi X\right)^{t}}\right]= & \frac{\Gamma_{m}(\alpha+\beta) \Gamma_{m}(\alpha+r) \Gamma_{m}(\beta+s)}{\Gamma_{m}(\alpha+\beta+r+s) \Gamma_{m}(\alpha) \Gamma_{m}(\beta)} \\
& \times \frac{{ }_{2} F_{1}(\alpha+r, \gamma+t ; \alpha+\beta+r+s ;-\Xi)}{{ }_{2} F_{1}(\alpha, \gamma ; \alpha+\beta ;-\Xi)},
\end{aligned}
$$

where $\operatorname{Re}(r)>-\alpha+(m-1) / 2$ and $\operatorname{Re}(s)>-\beta+(m-1) / 2$.

Proof. From the density of $X$,

$$
\begin{aligned}
& E\left[\frac{\operatorname{det}(X)^{r} \operatorname{det}\left(I_{m}-X\right)^{s}}{\operatorname{det}\left(I_{m}+\Xi X\right)^{t}}\right] \\
& \quad=C(\alpha, \beta, \gamma, \Xi) \int_{0}^{I_{m}} \frac{\operatorname{det}(X)^{\alpha+r-(m+1) / 2} \operatorname{det}\left(I_{m}-X\right)^{\beta+s-(m+1) / 2} d X}{\operatorname{det}\left(I_{m}+\Xi X\right)^{\gamma+t}} \\
& \quad=\frac{C(\alpha, \beta, \gamma, \Xi)}{C(\alpha+r, \beta+s, \gamma+t, \Xi)},
\end{aligned}
$$

where $\operatorname{Re}(r)>-\alpha+(m-1) / 2$ and $\operatorname{Re}(s)>-\beta+(m-1) / 2$. Simplifying this last expression using (3.2), we get the desired result.

From the density of $X$,

$$
E\left[C_{K}(X)\right]=C(\alpha, \beta, \gamma, \Xi) \int_{0}^{I_{m}} C_{\kappa}(X) \frac{\operatorname{det}(X)^{\alpha-(m+1) / 2} \operatorname{det}\left(I_{m}-X\right)^{\beta-(m+1) / 2} d X}{\operatorname{det}\left(I_{m}+\Xi X\right)^{\gamma}}
$$


For $\|\Xi\|<1$, writing $\operatorname{det}\left(I_{m}+\Xi X\right)^{-\gamma}$ in series involving zonal polynomials,

$$
\operatorname{det}\left(I_{m}+\Xi X\right)^{-\gamma}=\sum_{l=0}^{\infty} \sum_{\lambda \vdash l} \frac{(\gamma)_{\lambda}}{l !} C_{\lambda}(-\Xi X)
$$

and

$$
E\left[C_{\kappa}(X)\right]=C(\alpha, \beta, \gamma, \Xi) \sum_{l=0}^{\infty} \sum_{\lambda \vdash l} \frac{(\gamma)_{\lambda}}{l !} \Phi_{\kappa, \lambda}(\Xi),
$$

where

$$
\Phi_{\kappa, \lambda}(\Xi)=\int_{0}^{I_{m}} C_{\kappa}(X) C_{\lambda}(-\Xi X) \operatorname{det}(X)^{\alpha-(m+1) / 2} \operatorname{det}\left(I_{m}-X\right)^{\beta-(m+1) / 2} d X .
$$

Since $\Phi_{\kappa, \lambda}(\Xi)=\Phi_{\kappa, \lambda}\left(H^{T} \Xi H\right), H \in \mathrm{O}(m)$, integrating out $H$ in $\Phi_{\kappa, \lambda}\left(H^{T} \Xi H\right)$ using (2.4) and applying (2.7),

$$
\Phi_{\kappa, \lambda}(\Xi)=\frac{C_{\lambda}(-\Xi)}{C_{\lambda}\left(I_{m}\right)} \sum_{\phi \in \kappa \cdot \lambda}\left(\theta_{\phi}^{\kappa, \lambda}\right)^{2} \int_{0}^{I_{m}} C_{\phi}(X) \operatorname{det}(X)^{\alpha-(m+1) / 2} \operatorname{det}\left(I_{m}-X\right)^{\beta-(m+1) / 2} d X .
$$

Finally, integrating the above expression using (2.9) and substituting it in (4.10),

$$
E\left[C_{\kappa}(X)\right]=C(\alpha, \beta, \gamma, \Xi) \mathrm{B}_{m}(\alpha, \beta) \sum_{l=0}^{\infty} \sum_{\lambda \vdash l} \frac{(\gamma)_{\lambda}}{l !} \frac{C_{\lambda}(-\Xi)}{C_{\lambda}\left(I_{m}\right)} \sum_{\phi \in \kappa \cdot \lambda}\left(\theta_{\phi}^{\kappa, \lambda}\right)^{2} \frac{(\alpha)_{\phi}}{(\alpha+\beta)_{\phi}} C_{\phi}\left(I_{m}\right) .
$$

For $\|\Xi\|>1$, writing $\operatorname{det}\left(I_{m}+\Xi X\right)^{-\gamma}$ as

$$
\begin{aligned}
\operatorname{det}\left(I_{m}+\Xi X\right)^{-\gamma} & =\operatorname{det}\left(I_{m}+\Xi\right)^{-\gamma} \operatorname{det}\left(I_{m}-\left(I_{m}+\Xi\right)^{-1} \Xi\left(I_{m}-X\right)\right)^{-\gamma} \\
& =\operatorname{det}\left(I_{m}+\Xi\right)^{-\gamma} \sum_{l=0}^{\infty} \sum_{\lambda \vdash l} \frac{(\gamma)_{\lambda}}{l !} C_{\lambda}\left(\left(I_{m}+\Xi\right)^{-1} \Xi\left(I_{m}-X\right)\right)
\end{aligned}
$$

and following similar steps, we deduce that

$$
E\left[C_{\kappa}(X)\right]=C(\alpha, \beta, \gamma, \Xi) \operatorname{det}\left(I_{m}+\Xi\right)^{-\gamma} \sum_{l=0}^{\infty} \sum_{\lambda \vdash l} \frac{(\gamma)_{\lambda}}{l !} \Phi_{\kappa, \lambda}\left(\left(I_{m}+\Xi\right)^{-1} \Xi\right),
$$

where

$$
\begin{gathered}
\Phi_{\kappa, \lambda}\left(\left(I_{m}+\Xi\right)^{-1} \Xi\right)=\frac{C_{\lambda}\left(\left(I_{m}+\Xi\right)^{-1} \Xi\right)}{C_{\lambda}\left(I_{m}\right)} \sum_{\phi \in \kappa \cdot \lambda} \theta_{\phi}^{\kappa, \lambda} \int_{0}^{I_{m}} C_{\phi}^{\kappa, \lambda}\left(X, I_{m}-X\right) \\
\times \operatorname{det}(X)^{\alpha-(m+1) / 2} \operatorname{det}\left(I_{m}-X\right)^{\beta-(m+1) / 2} d X .
\end{gathered}
$$

Finally, integrating the above expression using (2.8) and substituting it in (4.11),

$$
\begin{aligned}
E\left[C_{\kappa}(X)\right]=C & (\alpha, \beta, \gamma, \Xi) \mathrm{B}_{m}(\alpha, \beta) \operatorname{det}\left(I_{m}+\Xi\right)^{-\gamma} \\
& \times \sum_{l=0}^{\infty} \sum_{\lambda \vdash l} \frac{(\gamma)_{\lambda}}{l !} \frac{C_{\lambda}\left(\left(I_{m}+\Xi\right)^{-1} \Xi\right)}{C_{\lambda}\left(I_{m}\right)} \sum_{\phi \in \kappa \cdot \lambda}\left(\theta_{\phi}^{\kappa, \lambda}\right)^{2} \frac{(\alpha)_{\kappa}(\beta)_{\lambda}}{(\alpha+\beta)_{\phi}} C_{\phi}\left(I_{m}\right) .
\end{aligned}
$$




\section{Quadratic forms}

In this section we obtain distributional results for the product of two independent random matrices involving the Gauss hypergeometric distribution.

Theorem 5.1. Suppose that $X_{1} \sim \mathrm{GH}_{m}\left(\alpha_{1}, \beta_{1}, \gamma_{1}, I_{m}\right)$ and $X_{2} \sim \mathrm{B} 1\left(m, \alpha_{2}, \beta_{2}\right)$ are independent. Then the p.d.f. of $Z=X_{2}^{1 / 2} X_{1} X_{2}^{1 / 2}$ is given by

$$
\begin{aligned}
& K_{1} \mathrm{~B}_{m}\left(\beta_{1}, \beta_{2}\right) \frac{\operatorname{det}(Z)^{\alpha_{1}-(m+1) / 2} \operatorname{det}\left(I_{m}-Z\right)^{\beta_{1}+\beta_{2}-(m+1) / 2}}{\operatorname{det}\left(I_{m}+Z\right)^{\gamma_{1}}} \\
& \quad \times F_{1}\left(\beta_{2}, \alpha_{1}+\beta_{1}-\alpha_{2}-\gamma_{1}, \gamma_{1} ; \beta_{1}+\beta_{2} ; I_{m}-Z,\left(I_{m}-Z\right)\left(I_{m}+Z\right)^{-1}\right),
\end{aligned}
$$

where $0<Z<I_{m}$ and

$$
K_{1}=\left\{\mathrm{B}_{m}\left(\alpha_{1}, \beta_{1}\right) \mathrm{B}_{m}\left(\alpha_{2}, \beta_{2}\right)_{2} F_{1}\left(\alpha_{1}, \gamma_{1} ; \alpha_{1}+\beta_{1} ;-I_{m}\right)\right\}^{-1} .
$$

Proof. Using the independence, the joint p.d.f. of $X_{1}$ and $X_{2}$ is given by

$$
\begin{aligned}
& K_{1} \frac{\operatorname{det}\left(X_{1}\right)^{\alpha_{1}-(m+1) / 2} \operatorname{det}\left(I_{m}-X_{1}\right)^{\beta_{1}-(m+1) / 2}}{\operatorname{det}\left(I_{m}+X_{1}\right)^{\gamma_{1}}} \\
& \quad \times \operatorname{det}\left(X_{2}\right)^{\alpha_{2}-(m+1) / 2} \operatorname{det}\left(I_{m}-X_{2}\right)^{\beta_{2}-(m+1) / 2},
\end{aligned}
$$

where $0<X_{i}<I_{m}, i=1,2$. Transforming $Z=X_{2}^{1 / 2} X_{1} X_{2}^{1 / 2}$ and $X_{2}=X_{2}$ with the Jacobian $J\left(X_{1}, X_{2} \rightarrow Z, X_{2}\right)=\operatorname{det}\left(X_{2}\right)^{-(m+1) / 2}$, we obtain the joint p.d.f. of $Z$ and $X_{2}$ :

$$
K_{1} \frac{\operatorname{det}(Z)^{\alpha_{1}-(m+1) / 2} \operatorname{det}\left(X_{2}-Z\right)^{\beta_{1}-(m+1) / 2} \operatorname{det}\left(I_{m}-X_{2}\right)^{\beta_{2}-(m+1) / 2}}{\operatorname{det}\left(X_{2}\right)^{\alpha_{1}+\beta_{1}-\left(\gamma_{1}+\alpha_{2}\right)} \operatorname{det}\left(X_{2}+Z\right)^{\gamma_{1}}},
$$

where $0<Z<X_{2}<I_{m}$. To find the marginal density of $Z$, we integrate (5.1) with respect to $X_{2}$ to get

$$
K_{1} \operatorname{det}(Z)^{\alpha_{1}-(m+1) / 2} \int_{Z}^{I_{m}} \frac{\operatorname{det}\left(X_{2}-Z\right)^{\beta_{1}-(m+1) / 2} \operatorname{det}\left(I_{m}-X_{2}\right)^{\beta_{2}-(m+1) / 2} d X_{2}}{\operatorname{det}\left(X_{2}\right)^{\alpha_{1}+\beta_{1}-\left(\gamma_{1}+\alpha_{2}\right)} \operatorname{det}\left(X_{2}+Z\right)^{\gamma_{1}}} .
$$

In (5.2), the change of variables $W=\left(I_{m}-Z\right)^{-1 / 2}\left(I_{m}-X_{2}\right)\left(I_{m}-Z\right)^{-1 / 2}$ yields

$$
\begin{aligned}
& K_{1} \frac{\operatorname{det}(Z)^{\alpha_{1}-(m+1) / 2} \operatorname{det}\left(I_{m}-Z\right)^{\beta_{1}+\beta_{2}-(m+1) / 2}}{\operatorname{det}\left(I_{m}+Z\right)^{\gamma_{1}}} \\
& \quad \times \int_{0}^{I_{m}} \frac{\operatorname{det}(W)^{\beta_{2}-(m+1) / 2} \operatorname{det}\left(I_{m}-W\right)^{\beta_{1}-(m+1) / 2} d W}{\operatorname{det}\left(I_{m}-\left(I_{m}-Z\right) W\right)^{\alpha_{1}+\beta_{1}-\gamma_{1}-\alpha_{2}} \operatorname{det}\left(I_{m}-\left(I_{m}-Z\right)^{1 / 2}\left(I_{m}+Z\right)^{-1}\left(I_{m}-Z\right)^{1 / 2} W\right)^{\gamma_{1}}} .
\end{aligned}
$$

Finally, noting that

$$
\left(I_{m}-Z\right)^{1 / 2}\left(I_{m}+Z\right)^{-1}\left(I_{m}-Z\right)^{1 / 2}=\left(I_{m}-Z\right)\left(I_{m}+Z\right)^{-1}=\left(I_{m}+Z\right)^{-1}\left(I_{m}-Z\right)
$$

and applying (2.10), we obtain the desired result. 
Corollary 5.2. Suppose that $X_{1} \sim \mathrm{B} 1\left(m, \alpha_{1}, \beta_{1}\right)$ and $X_{2} \sim \mathrm{B} 1\left(m, \alpha_{2}, \beta_{2}\right)$ are independent. Then the p.d.f. of $Z=X_{2}^{1 / 2} X_{1} X_{2}^{1 / 2}$, for $0<Z<I_{m}$, is given by

$$
\begin{aligned}
& \frac{\mathrm{B}_{m}\left(\beta_{1}, \beta_{2}\right)}{\mathrm{B}_{m}\left(\alpha_{1}, \beta_{1}\right) \mathrm{B}_{m}\left(\alpha_{2}, \beta_{2}\right)} \operatorname{det}(Z)^{\alpha_{1}-(m+1) / 2} \operatorname{det}\left(I_{m}-Z\right)^{\beta_{1}+\beta_{2}-(m+1) / 2} \\
& \quad \times{ }_{2} F_{1}\left(\beta_{2}, \alpha_{1}+\beta_{1}-\alpha_{2} ; \beta_{1}+\beta_{2} ; I_{m}-Z\right) .
\end{aligned}
$$

Further, if $\alpha_{1}+\beta_{1}=\alpha_{2}$, then $X_{2}^{1 / 2} X_{1} X_{2}^{1 / 2} \sim \mathrm{B} 1\left(m, \alpha_{1}, \beta_{1}+\beta_{2}\right)$.

Next, we give the density of $Z=X_{2}^{1 / 2} X_{1} X_{2}^{1 / 2}$, where $X_{1} \sim \mathrm{GH}_{m}\left(\alpha_{1}, \beta_{1}, \gamma_{1}, \Xi_{1}\right)$ and $X_{2} \sim \mathrm{B} 3\left(m, \alpha_{2}, \beta_{2}\right)$. We represent this density in terms of Appell's first hypergeometric function $F_{1}$ of three $m \times m$ symmetric matrix arguments, which is defined by

$$
\begin{aligned}
& F_{1}\left(a, b_{1}, b_{2}, b_{3} ; c ; Z_{1}, Z_{2}, Z_{3}\right) \\
& \quad=\frac{\Gamma_{m}(c)}{\Gamma_{m}(a) \Gamma_{m}(c-a)} \int_{0}^{I_{m}} \frac{\operatorname{det}(V)^{a-(m+1) / 2} \operatorname{det}\left(I_{m}-V\right)^{c-a-(m+1) / 2} d V}{\operatorname{det}\left(I_{m}-V Z_{1}\right)^{b_{1}} \operatorname{det}\left(I_{m}-V Z_{2}\right)^{b_{2} \operatorname{det}\left(I_{m}-V Z_{3}\right)^{b_{3}}} .} .
\end{aligned}
$$

Note that $F_{1}\left(a, b_{1}, b_{2}, 0 ; c ; Z_{1}, Z_{2}, Z_{3}\right)=F_{1}\left(a, b_{1}, b_{2} ; c ; Z_{1}, Z_{2}\right)$.

Theorem 5.3. Suppose that $X_{1} \sim \mathrm{GH}_{m}\left(\alpha_{1}, \beta_{1}, \gamma_{1}, I_{m}\right)$ and $X_{2} \sim \mathrm{B} 3\left(m, \alpha_{2}, \beta_{2}\right)$ are independent. Then the p.d.f. of $Z=X_{2}^{1 / 2} X_{1} X_{2}^{1 / 2}$ is

$$
\begin{gathered}
K_{1} \mathrm{~B}_{m}\left(\beta_{1}, \beta_{2}\right) \frac{\operatorname{det}(Z)^{\alpha_{1}-(m+1) / 2} \operatorname{det}\left(I_{m}-Z\right)^{\beta_{1}+\beta_{2}-(m+1) / 2}}{2^{m \beta_{2}} \operatorname{det}\left(I_{m}+Z\right)^{\gamma_{1}}} \\
\times F_{1}\left(\beta_{2}, \alpha_{1}+\beta_{1}-\alpha_{2}-\gamma_{1}, \alpha_{2}+\beta_{2}, \gamma_{1} ; \beta_{1}+\beta_{2} ;\right. \\
\left.I_{m}-Z, \frac{I_{m}-Z}{2},\left(I_{m}-Z\right)\left(I_{m}+Z\right)^{-1}\right),
\end{gathered}
$$

where $0<Z<I_{m}$ and $K_{1}$ is defined in Theorem 5.1.

Proof. Using the independence, the joint p.d.f. of $X_{1}$ and $X_{2}$ is given by

$$
\begin{array}{r}
2^{m \alpha_{2}} K_{1} \frac{\operatorname{det}\left(X_{1}\right)^{\alpha_{1}-(m+1) / 2} \operatorname{det}\left(I_{m}-X_{1}\right)^{\beta_{1}-(m+1) / 2}}{\operatorname{det}\left(I_{m}+X_{1}\right)^{\gamma_{1}}} \\
\times \frac{\operatorname{det}\left(X_{2}\right)^{\alpha_{2}-(m+1) / 2} \operatorname{det}\left(I_{m}-X_{2}\right)^{\beta_{2}-(m+1) / 2}}{\operatorname{det}\left(I_{m}+X_{2}\right)^{\alpha_{2}+\beta_{2}}},
\end{array}
$$

where $0<X_{i}<I_{m}$ when $i=1,2$. Transforming $Z=X_{2}^{1 / 2} X_{1} X_{2}^{1 / 2}$ and $X_{2}=X_{2}$, with Jacobian $J\left(X_{1}, X_{2} \rightarrow Z, X_{2}\right)=\operatorname{det}\left(X_{2}\right)^{-(m+1) / 2}$, we obtain the joint p.d.f. of $Z$ and $X_{2}$ as

$$
2^{m \alpha_{2}} K_{1} \frac{\operatorname{det}(Z)^{\alpha_{1}-(m+1) / 2} \operatorname{det}\left(X_{2}-Z\right)^{\beta_{1}-(m+1) / 2} \operatorname{det}\left(I_{m}-X_{2}\right)^{\beta_{2}-(m+1) / 2}}{\operatorname{det}\left(X_{2}\right)^{\alpha_{1}+\beta_{1}-\left(\gamma_{1}+\alpha_{2}\right)} \operatorname{det}\left(X_{2}+Z\right)^{\gamma_{1}} \operatorname{det}\left(I_{m}+X_{2}\right)^{\alpha_{2}+\beta_{2}}},
$$


where $0<Z<X_{2}<I_{m}$. To find the marginal p.d.f. of $Z$, we integrate (5.4) with respect to $X_{2}$ to get

$$
\begin{aligned}
& 2^{m \alpha_{2}} K_{1} \operatorname{det}(Z)^{\alpha_{1}-(m+1) / 2} \int_{Z}^{I_{m}} \frac{\operatorname{det}\left(X_{2}-Z\right)^{\beta_{1}-(m+1) / 2} \operatorname{det}\left(I_{m}-X_{2}\right)^{\beta_{2}-(m+1) / 2} d X_{2}}{\operatorname{det}\left(X_{2}\right)^{\alpha_{1}+\beta_{1}-\left(\gamma_{1}+\alpha_{2}\right)} \operatorname{det}\left(X_{2}+Z\right)^{\gamma_{1}} \operatorname{det}\left(I_{m}+X_{2}\right)^{\alpha_{2}+\beta_{2}}} \\
&=2^{-m \beta_{2}} K_{1} \frac{\operatorname{det}(Z)^{\alpha_{1}-(m+1) / 2} \operatorname{det}\left(I_{m}-Z\right)^{\beta_{1}+\beta_{2}-(m+1) / 2}}{\operatorname{det}\left(I_{m}+Z\right)^{\gamma_{1}}} \\
& \times \int_{0}^{I_{m}} \frac{\operatorname{det}(W)^{\beta_{2}-(m+1) / 2} \operatorname{det}\left(I_{m}-W\right)^{\beta_{1}-(m+1) / 2}}{\operatorname{det}\left(I_{m}-\left(I_{m}-Z\right)^{1 / 2}\left(I_{m}+Z\right)^{-1}\left(I_{m}-Z\right)^{1 / 2} W\right)^{\gamma_{1}}} \\
& \quad \times \frac{\operatorname{det}\left(I_{m}-\left(I_{m}-Z\right) W\right)^{\gamma_{1}+\alpha_{2}-\alpha_{1}-\beta_{1}} d W}{\operatorname{det}\left(I_{m}-\left(I_{m}-Z\right) W / 2\right)^{\alpha_{2}+\beta_{2}}},
\end{aligned}
$$

where we have used the substitution $W=\left(I_{m}-Z\right)^{-1 / 2}\left(I_{m}-X_{2}\right)\left(I_{m}-Z\right)^{-1 / 2}$. Finally, observing that

$$
\left(I_{m}-Z\right)^{1 / 2}\left(I_{m}+Z\right)^{-1}\left(I_{m}-Z\right)^{1 / 2}=\left(I_{m}-Z\right)\left(I_{m}+Z\right)^{-1}=\left(I_{m}+Z\right)^{-1}\left(I_{m}-Z\right)
$$

and applying (5.3), we obtain the desired result.

Corollary 5.4. Suppose that $X_{1} \sim \mathrm{B} 1\left(m, \alpha_{1}, \beta_{1}\right)$ and $X_{2} \sim \mathrm{B} 3\left(m, \alpha_{2}, \beta_{2}\right)$ are independent. Then the p.d.f. of $Z=X_{2}^{1 / 2} X_{1} X_{2}^{1 / 2}$ is given by

$$
\begin{aligned}
& \frac{2^{-m \beta_{2}} \Gamma_{m}\left(\alpha_{1}+\beta_{1}\right) \Gamma_{m}\left(\alpha_{2}+\beta_{2}\right)}{\Gamma_{m}\left(\alpha_{1}\right) \Gamma_{m}\left(\alpha_{2}\right) \Gamma_{m}\left(\beta_{1}+\beta_{2}\right)} \operatorname{det}(Z)^{\alpha_{1}-(m+1) / 2} \operatorname{det}\left(I_{m}-Z\right)^{\beta_{1}+\beta_{2}-(m+1) / 2} \\
& \quad \times F_{1}\left(\beta_{2}, \alpha_{1}+\beta_{1}-\alpha_{2}, \alpha_{2}+\beta_{2}, \beta_{1}+\beta_{2} ; I_{m}-Z, \frac{I_{m}-Z}{2}\right),
\end{aligned}
$$

where $0<Z<I_{m}$. Further, if $\alpha_{2}=\alpha_{1}+\beta_{1}$, then the p.d.f. of $Z=X_{2}^{1 / 2} X_{1} X_{2}^{1 / 2}$, for $0<Z<I_{m}$, is given by

$$
\begin{aligned}
& \frac{2^{-m \beta_{2}} \Gamma_{m}\left(\alpha_{1}+\beta_{1}+\beta_{2}\right)}{\Gamma_{m}\left(\alpha_{1}\right) \Gamma_{m}\left(\beta_{1}+\beta_{2}\right)} \operatorname{det}(Z)^{\alpha_{1}-(m+1) / 2} \operatorname{det}\left(I_{m}-Z\right)^{\beta_{1}+\beta_{2}-(m+1) / 2} \\
& \quad \times{ }_{2} F_{1}\left(\beta_{2}, \alpha_{1}+\beta_{1}+\beta_{2} ; \beta_{1}+\beta_{2} ; \frac{I_{m}-Z}{2}\right) .
\end{aligned}
$$

Theorem 5.5. Suppose that $X_{1} \sim \mathrm{GH}_{m}\left(\alpha_{1}, \beta_{1}, \gamma_{1}, \Xi_{1}\right)$ and $X_{2} \sim \mathrm{B} 2\left(m, \alpha_{2}, \beta_{2}\right)$ are independent. Then the p.d.f. of $Z=X_{1}^{1 / 2} X_{2} X_{1}^{1 / 2}$ is given by

$$
\begin{aligned}
& K_{1}\left(\Xi_{1}\right) \frac{\mathrm{B}_{m}\left(\beta_{1}, \alpha_{1}+\beta_{2}\right)}{\operatorname{det}\left(I_{m}+\Xi_{1}\right)^{\gamma_{1}}} \frac{\operatorname{det}(Z)^{\alpha_{2}-(m+1) / 2}}{\operatorname{det}\left(I_{m}+Z\right)^{\alpha_{2}+\beta_{2}}} \\
& \quad \times F_{1}\left(\beta_{1}, \gamma_{1}, \alpha_{2}+\beta_{2} ; \alpha_{1}+\beta_{1}+\beta_{2} ;\left(I_{m}+\Xi_{1}\right)^{-1} \Xi_{1},\left(I_{m}+Z\right)^{-1}\right),
\end{aligned}
$$

where $Z>0$ and $K_{1}\left(\Xi_{1}\right)$ is defined by

$$
K_{1}\left(\Xi_{1}\right)=\left\{\mathrm{B}_{m}\left(\alpha_{1}, \beta_{1}\right) \mathrm{B}_{m}\left(\alpha_{2}, \beta_{2}\right)_{2} F_{1}\left(\alpha_{1}, \gamma_{1} ; \alpha_{1}+\beta_{1} ;-\Xi_{1}\right)\right\}^{-1} .
$$


Proof. Since $X_{1}$ and $X_{2}$ are independent, their joint p.d.f. is given by

$$
K_{1}\left(\Xi_{1}\right) \frac{\operatorname{det}\left(X_{1}\right)^{\alpha_{1}-(m+1) / 2} \operatorname{det}\left(I_{m}-X_{1}\right)^{\beta_{1}-(m+1) / 2} \operatorname{det}\left(X_{2}\right)^{\alpha_{2}-(m+1) / 2}}{\operatorname{det}\left(I_{m}+\Xi_{1} X_{1}\right)^{\gamma_{1}} \operatorname{det}\left(I_{m}+X_{2}\right)^{\alpha_{2}+\beta_{2}}},
$$

where $0<X_{1}<I_{m}$ and $X_{2}>0$. Now consider the transformation $Z=X_{1}^{1 / 2} X_{2} X_{1}^{1 / 2}$ and $W=I_{m}-X_{1}$, whose Jacobian is $J\left(X_{1}, X_{2} \rightarrow W, Z\right)=\operatorname{det}\left(I_{m}-W\right)^{-(m+1) / 2}$. Thus, we obtain the joint p.d.f. of $W$ and $Z$ as

$$
\begin{aligned}
K_{1}\left(\Xi_{1}\right) & \frac{\operatorname{det}(Z)^{\alpha_{2}-(m+1) / 2}}{\operatorname{det}\left(I_{m}+\Xi_{1}\right)^{\gamma_{1}} \operatorname{det}\left(I_{m}+Z\right)^{\alpha_{2}+\beta_{2}}} \\
& \times \frac{\operatorname{det}(W)^{\beta_{1}-(m+1) / 2} \operatorname{det}\left(I_{m}-W\right)^{\alpha_{1}+\beta_{2}-(m+1) / 2}}{\operatorname{det}\left(I_{m}-\left(I_{m}+\Xi_{1}\right)^{-1} \Xi_{1} W\right)^{\gamma_{1}} \operatorname{det}\left(I_{m}-\left(I_{m}+Z\right)^{-1} W\right)^{\alpha_{2}+\beta_{2}}},
\end{aligned}
$$

where $0<W<I_{m}$. Finally, integrating $W$ using (2.10), we obtain the desired result.

Corollary 5.6. Suppose that $X_{1} \sim \mathrm{B} 1\left(m, \alpha_{1}, \beta_{1} ; \Lambda_{1}\right)$ and $X_{2} \sim \mathrm{B} 2\left(m, \alpha_{2}, \beta_{2}\right)$ are independent. Then the p.d.f. of $Z=X_{1}^{1 / 2} X_{2} X_{1}^{1 / 2}$, for $Z>0$, is given by

$$
\begin{aligned}
& \frac{\mathrm{B}_{m}\left(\beta_{1}, \alpha_{1}+\beta_{2}\right)}{\operatorname{det}\left(\Lambda_{1}\right)^{\beta_{1}} \mathrm{~B}_{m}\left(\alpha_{1}, \beta_{1}\right) \mathrm{B}_{m}\left(\alpha_{2}, \beta_{2}\right)} \frac{\operatorname{det}(Z)^{\alpha_{2}-(m+1) / 2}}{\operatorname{det}\left(I_{m}+Z\right)^{\alpha_{2}+\beta_{2}}} \\
& \quad \times F_{1}\left(\beta_{1}, \alpha_{1}+\beta_{1}, \alpha_{2}+\beta_{2} ; \alpha_{1}+\beta_{1}+\beta_{2} ; I_{m}-\Lambda_{1}^{-1},\left(I_{m}+Z\right)^{-1}\right) .
\end{aligned}
$$

Corollary 5.7. Suppose that $X_{1} \sim \mathrm{B} 1\left(m, \alpha_{1}, \beta_{1}\right)$ and $X_{2} \sim \mathrm{B} 2\left(m, \alpha_{2}, \beta_{2}\right)$ are independent. Then the p.d.f. of $Z=X_{1}^{1 / 2} X_{2} X_{1}^{1 / 2}$, for $Z>0$, is given by

$$
\frac{\mathrm{B}_{m}\left(\beta_{1}, \alpha_{1}+\beta_{2}\right)}{\mathrm{B}_{m}\left(\alpha_{1}, \beta_{1}\right) \mathrm{B}_{m}\left(\alpha_{2}, \beta_{2}\right)} \frac{\operatorname{det}(Z)^{\alpha_{2}-(m+1) / 2}}{\operatorname{det}\left(I_{m}+Z\right)^{\alpha_{2}+\beta_{2}}}{ }_{2} F_{1}\left(\beta_{1}, \alpha_{2}+\beta_{2} ; \alpha_{1}+\beta_{1}+\beta_{2} ;\left(I_{m}+Z\right)^{-1}\right) \text {. }
$$

Further, if $\alpha_{2}=\alpha_{1}+\beta_{1}$, then $X_{1}^{1 / 2} X_{2} X_{1}^{1 / 2} \sim \mathrm{B} 2\left(m, \alpha_{1}, \beta_{2}\right)$.

Corollary 5.8. Suppose that $X_{1} \sim \mathrm{B} 3\left(m, \alpha_{1}, \beta_{1}\right)$ and $X_{2} \sim \mathrm{B} 2\left(m, \alpha_{2}, \beta_{2}\right)$ are independent. Then the p.d.f. of $Z=X_{1}^{1 / 2} X_{2} X_{1}^{1 / 2}$, for $Z>0$, is given by

$$
\begin{aligned}
& \frac{2^{-m \beta_{1}} \mathrm{~B}_{m}\left(\beta_{1}, \alpha_{1}+\beta_{2}\right)}{\mathrm{B}_{m}\left(\alpha_{1}, \beta_{1}\right) \mathrm{B}_{m}\left(\alpha_{2}, \beta_{2}\right)} \frac{\operatorname{det}(Z)^{\alpha_{2}-(m+1) / 2}}{\operatorname{det}\left(I_{m}+Z\right)^{\alpha_{2}+\beta_{2}}} \\
& \quad \times F_{1}\left(\beta_{1}, \alpha_{1}+\beta_{1}, \alpha_{2}+\beta_{2} ; \alpha_{1}+\beta_{1}+\beta_{2} ; 2^{-1} I_{m},\left(I_{m}+Z\right)^{-1}\right) .
\end{aligned}
$$

In the next theorem we derive the density function of $Z_{1}=X^{-1 / 2} Y X^{-1 / 2}$, where the random matrices $X$ and $Y$ are independent, $X \sim \mathrm{GH}_{m}(\alpha, \beta, \gamma, \Xi)$ and the distribution of $Y$ is matrix-variate gamma. An $m \times m$ random symmetric positive definite matrix $Y$ is said to have a matrix-variate gamma distribution with parameters $\Psi$ and $\kappa$, denoted by $Y \sim \mathrm{Ga}(m, \kappa, \Psi)$, if its p.d.f. is given by

$$
\frac{\operatorname{etr}\left(-\Psi^{-1} Y\right) \operatorname{det}(Y)^{\kappa-(m+1) / 2}}{\Gamma_{m}(\kappa) \operatorname{det}(\Psi)^{\kappa}}
$$

where $Y>0, \Psi>0$, and $\kappa>(m-1) / 2$. 
Theorem 5.9. Suppose that the random matrices $X$ and $Y$ are independent, and that $X \sim \mathrm{GH}_{m}(\alpha, \beta, \gamma, \Xi)$ and $Y \sim \mathrm{Ga}\left(m, \kappa, I_{m}\right)$. Then the p.d.f. of $Z_{1}=X^{-1 / 2} Y X^{-1 / 2}$ is given by

$$
K_{2} \frac{\mathbf{B}_{m}(\alpha+\kappa, \beta) \operatorname{det}\left(Z_{1}\right)^{\kappa-(m+1) / 2} \operatorname{etr}\left(-Z_{1}\right)}{\operatorname{det}\left(I_{m}+\Xi\right)^{\gamma}} \boldsymbol{\Phi}_{1}\left(\beta, \gamma ; \alpha+\beta+\kappa ;\left(I_{m}+\Xi\right)^{-1} \Xi, Z_{1}\right),
$$

where $Z_{1}>0$ and

$$
K_{2}=\left\{\Gamma_{m}(\kappa) \mathrm{B}_{m}(\alpha, \beta)_{2} F_{1}(\alpha, \gamma ; \alpha+\beta ;-\Xi)\right\}^{-1} .
$$

Proof. The joint p.d.f. of $X$ and $Y$ is given by

$$
K_{2} \frac{\operatorname{det}(X)^{\alpha-(m+1) / 2} \operatorname{det}\left(I_{m}-X\right)^{\beta-(m+1) / 2} \operatorname{det}(Y)^{\kappa-(m+1) / 2}}{\operatorname{det}\left(I_{m}+\Xi X\right)^{\gamma} \operatorname{etr}(Y)},
$$

where $0<X<I_{m}$ and $Y>0$. Now, transforming $Z_{1}=X^{-1 / 2} Y X^{-1 / 2}$ and $W=I_{m}-X$, with the Jacobian $J\left(X, Y \rightarrow W, Z_{1}\right)=\operatorname{det}\left(I_{m}-W\right)^{(m+1) / 2}$, we obtain the joint p.d.f. of $Z_{1}$ and $W$ as

$$
K_{2} \frac{\operatorname{etr}\left(-Z_{1}\right) \operatorname{det}\left(Z_{1}\right)^{\kappa-(m+1) / 2}}{\operatorname{det}\left(I_{m}+\Xi\right)^{\gamma}} \frac{\operatorname{det}(W)^{\beta-(m+1) / 2} \operatorname{det}\left(I_{m}-W\right)^{\alpha+\kappa-(m+1) / 2}}{\operatorname{det}\left(I_{m}-\left(I_{m}+\Xi\right)^{-1} \Xi W\right)^{\gamma} \operatorname{etr}\left(-W Z_{1}\right)},
$$

where $0<W<I_{m}$ and $Z_{1}>0$. Now, integrating $W$ using (2.11), we get the marginal density of $Z_{1}$.

Corollary 5.10. Suppose that the random matrices $X$ and $Y$ are independent, and that $X \sim \mathrm{B} 3(m, \alpha, \beta)$ and $Y \sim \mathrm{Ga}\left(m, \kappa, I_{m}\right)$. Then the p.d.f. of $Z_{1}=X^{-1 / 2} Y X^{-1 / 2}$ is given by

$$
\frac{\Gamma_{m}(\alpha+\kappa) \Gamma_{m}(\alpha+\beta) \operatorname{det}\left(Z_{1}\right)^{\kappa-(m+1) / 2} \operatorname{etr}\left(-Z_{1}\right)}{2^{m \beta} \Gamma_{m}(\kappa) \Gamma_{m}(\alpha) \Gamma_{m}(\alpha+\beta+\kappa)} \boldsymbol{\Phi}_{1}\left(\beta, \alpha+\beta ; \alpha+\beta+\kappa ; \frac{I_{m}}{2}, Z_{1}\right),
$$

where $Z_{1}>0$.

Corollary 5.11. Suppose that the random matrices $X$ and $Y$ are independent, and that $X \sim \mathrm{B} 1(m, \alpha, \beta)$ and $Y \sim \mathrm{Ga}\left(m, \kappa, I_{m}\right)$. Then the p.d.f. of $Z_{1}=X^{-1 / 2} Y X^{-1 / 2}$ is given by

$$
\frac{\Gamma_{m}(\alpha+\kappa) \Gamma_{m}(\alpha+\beta) \operatorname{det}\left(Z_{1}\right)^{\kappa-(m+1) / 2} \operatorname{etr}\left(-Z_{1}\right)}{\Gamma_{m}(\kappa) \Gamma_{m}(\alpha) \Gamma_{m}(\alpha+\beta+\kappa)}{ }_{1} F_{1}\left(\beta ; \alpha+\beta+\kappa ; Z_{1}\right),
$$

where $Z_{1}>0$.

\section{Acknowledgement}

The authors wish to thank the referee for many useful comments. 


\section{References}

[1] T. W. Anderson, An Introduction to Multivariate Statistical Analysis, 3rd edn, Wiley Series in Probability and Statistics (John Wiley \& Sons, Hoboken, NJ, 2003).

[2] C. Armero and M. J. Bayarri, 'Prior assessments for predictions in queues', The Statistician 43(1) (1994), 139-153.

[3] L. Cardeño, D. K. Nagar and L. E. Sánchez, 'Beta type 3 distribution and its multivariate generalization', Tamsui Oxf. J. Math. Sci. 21(2) (2005), 225-241.

[4] Y. Chikuse, 'Distributions of some matrix variates and latent roots in multivariate Behrens-Fisher discriminant analysis', Ann. Statist. 9(2) (1981), 401-407.

[5] A. G. Constantine, 'Some noncentral distribution problems in multivariate analysis', Ann. Math. Statist. 34 (1963), 1270-1285.

[6] A. W. Davis, 'Invariant polynomials with two matrix arguments extending the zonal polynomials: applications to multivariate distribution theory', Ann. Inst. Statist. Math. 31(3) (1979), 465-485.

[7] A. W. Davis, 'Invariant polynomials with two matrix arguments, extending the zonal polynomials', in: Multivariate Analysis, Vol. V (Proc. Fifth Internat. Sympos., Univ. Pittsburgh, Pittsburgh, PA, 1978) (North-Holland, Amsterdam, 1980), pp. 287-299.

[8] A. K. Gupta and D. K. Nagar, Matrix Variate Distributions, Chapman \& Hall/CRC Monographs and Surveys in Pure and Applied Mathematics, 104 (Chapman \& Hall/CRC, Boca Raton, FL, 2000).

[9] A. K. Gupta and D. K. Nagar, 'Properties of matrix variate beta type 3 distribution', Int. J. Math. Math. Sci. 2009 (2009), p. 18, Art. ID 308518.

[10] C. S. Herz, 'Bessel functions of matrix argument', Ann. of Math. (2) 61 (1955), 474-523.

[11] A. T. James, 'Distributions of matrix variates and latent roots derived from normal samples', Ann. Math. Statist. 35 (1964), 475-501.

[12] N. L. Johnson, S. Kotz and N. Balakrishnan, Continuous Univariate Distributions, Vol. 2, 2nd edn, Wiley Series in Probability and Mathematical Statistics: Applied Probability and Statistics (John Wiley \& Sons, New York, 1995).

[13] D. L. Libby and M. R. Novic, 'Multivariate generalized beta distributions with applications to utility assessment', J. Educ. Statist. 35 (1982), 271-294.

[14] Y. L. Luke, The Special Functions and their Approximations, Vol. I, Mathematics in Science and Engineering, 53 (Academic Press, New York, 1969).

[15] S. Nadarajah, 'Sums, products and ratios of generalized beta variables', Statist. Papers 47(1) (2006), 69-90.

[16] D. K. Nagar and A. K. Gupta, 'Matrix-variate Kummer-beta distribution', J. Aust. Math. Soc. 73(1) (2002), 11-25.

[17] D. K. Nagar and E. A. Rada-Mora, 'Properties of multivariate beta distributions', Far East J. Theor. Stat. 24(1) (2008), 73-94.

[18] R. K. Saxena, P. L. Sethi and O. P. Gupta, 'Appell functions of matrix arguments', Indian J. Pure Appl. Math. 28(3) (1997), 371-380.

[19] M. S. Srivastava and C. G. Khatri, An Introduction to Multivariate Statistics (North-Holland, New York, 1979).

\section{ARJUN K. GUPTA, Department of Mathematics and Statistics, Bowling Green State University, Bowling Green, OH 43403-0221, USA e-mail: gupta@bgnet.bgsu.edu}

DAYA K. NAGAR, Instituto de Matemáticas, Universidad de Antioquia, Calle 67, No. 53-108, Medellín, Colombia

e-mail: dayaknagar@yahoo.com 